\title{
Alkene ozonolysis in the presence of diazo functionality: accessing an intermediate for squalestatin synthesis
}

\author{
Hasanain A. A. Almohsenia,1 \\ Younes Fegheh-Hassanpour \\ Tanzeel Arif \\ David M. Hodgson*a \\ a Department of Chemistry, Chemistry Research Laboratory, \\ University of Oxford, Mansfield Road, Oxford OX1 3TA, United \\ Kingdom. \\ david.hodgson@chem.ox.ac.uk
}

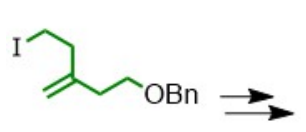

$\mathrm{EtO}_{2} \mathrm{C} \mathrm{N}_{2}$

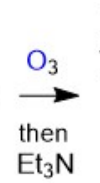

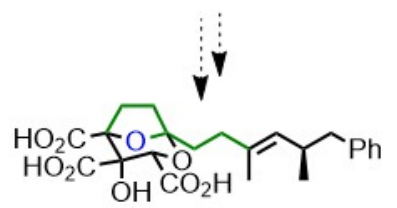

Abstract Studies on both the propensity for intramolecular cycloaddition between diazo and alkene functionality, and the tolerance of $\alpha$-substituted $\alpha$-diazoesters towards ozone in the presence of an alkene, led to chemoselective alkene ozonolysis of an $\varepsilon$-unsaturated- $\alpha$-diazoester to give a key racemic diazoketone for the synthesis of 6,7-dideoxysqualestatin H5.

Key words 6,7-dideoxysqualestatin $\mathrm{H} 5$, squalestatin synthesis, alkene ozonolysis, intramolecular cycloaddition, diazo stability, pyrazoline.

\section{Introduction}

We recently reported two syntheses of 6,7-dideoxysqualestatin H5 (DDSQ) 7 (Scheme 1).2,3 Both syntheses proceeded through diazoketones 4, which underwent rhodium(II)-catalysed tandem carbonyl ylide formation-intermolecular dipolar cycloadditions $(\mathbf{4} \rightarrow \mathbf{5} \rightarrow \mathbf{6}), 4$ followed by acid-catalysed rearrangement to generate the characteristic 2,8-dioxabicyclo [3.2.1] octane core of the squalestatins / zaragozic acids at the correct tricarboxylic acid oxidation level. Also in both cases, the silyloxyester stereocentre was created by stereoselective

\begin{abstract}
alkylation of dimethyl tartrate acetonide (8), with 5-6 steps being subsequently required to produce the diazoketones 4 . In principle, a more concise approach to such cycloaddition precursors 4 could proceed by aldol reaction between a diazoacetate anion $\mathbf{2}$ and an unsaturated $\alpha$-ketoester $\mathbf{1}$, followed by chemoselective alkene ozonolysis of the resulting unsaturated diazodiester 3 (Scheme 1). Here we report the realisation of this latter strategy, despite concerns over both intrinsic instability of the aldol product to spontaneous intramolecular cycloaddition between the diazo and alkene functionality, and of undesired conversion of diazo into keto functionality by ozone.
\end{abstract}

\section{Results and discussion}

Before embarking on the above route $(\mathbf{1} \rightarrow \mathbf{3} \rightarrow \mathbf{4})$, it was considered prudent to first establish on simpler substrates the proclivity of $\alpha$-diazoester functionality to engage in intramolecular cycloaddition with an electron-rich alkene to form bicyclic pyrazolines. 5

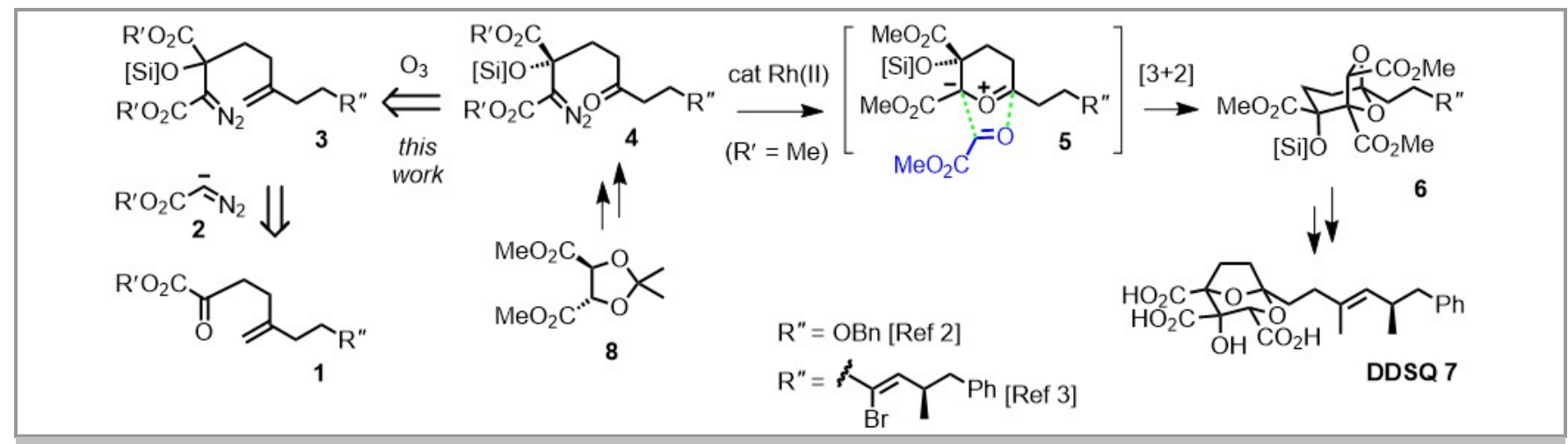

Scheme 1 Approaches to 6,7-dideoxysqualestatin H5 (7). 
Unsaturated hydrazones 9a, $\mathbf{b}^{6}$ underwent $\mathrm{Et}_{3} \mathrm{~N}$-induced diazo formation ${ }^{7}$ in $\mathrm{CH}_{2} \mathrm{Cl}_{2}$ at $\mathrm{rt}$ (Scheme 2). As anticipated from Dauben's studies, $5 \mathrm{~b}$ this led hydrazone 9a to form 1-pyrazoline 11a, in 92\% yield (Scheme 2). However, intramolecular cycloaddition was minimised with the 2,2-dialkyl-substituted terminal alkene $\mathbf{9 b}$, to give a mixture (52\%) mainly consisting of unsaturated diazoester 10b together with only small amounts of bicycle 11b (10b:11b, 91:9).

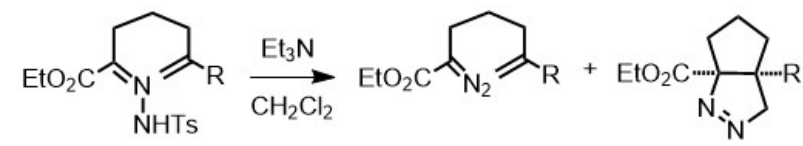

$$
\begin{aligned}
& \text { 9a }(\mathrm{R}=\mathrm{H}) \\
& 9 \mathrm{~b}(\mathrm{R}=\mathrm{Me}) \\
& 10 b(R=M e) \\
& 11 \mathrm{a}(\mathrm{R}=\mathrm{H}) \\
& \text { 11b }(\mathrm{R}=\mathrm{Me})
\end{aligned}
$$

Scheme 2 Propensity for diazoalkenes to undergo intramolecular cycloaddition.

The stability of the $\alpha$-diazoester motif towards ozone was next examined. The diazo moiety could be oxidised to a carbonyl group by ozone.6,8 Model compounds 13a,b (Scheme 3), that contain the diazo moiety in a similar environment to diazodiester 3 (Scheme 1), were prepared by aldol reaction between ethyl pyruvate (12) and the anion of ethyl diazoacetate (2), ${ }^{9}$ followed by silylation. Equimolar solutions of $\alpha$-diazoesters $\mathbf{1 3 a} \mathbf{a} \mathbf{b}$ and alkene $\mathbf{1 4}$, the latter mimicking the alkene portion of 3 , were exposed to ozone at $-78{ }^{\circ} \mathrm{C}_{\text {in }} \mathrm{CH}_{2} \mathrm{Cl}_{2}$, followed by addition of $\mathrm{Et}_{3} \mathrm{~N} .10$ Pleasingly, these conditions gave ketone 15, with diazoesters 13a,b being recovered intact (Scheme 3, see also SI: Figure S1).

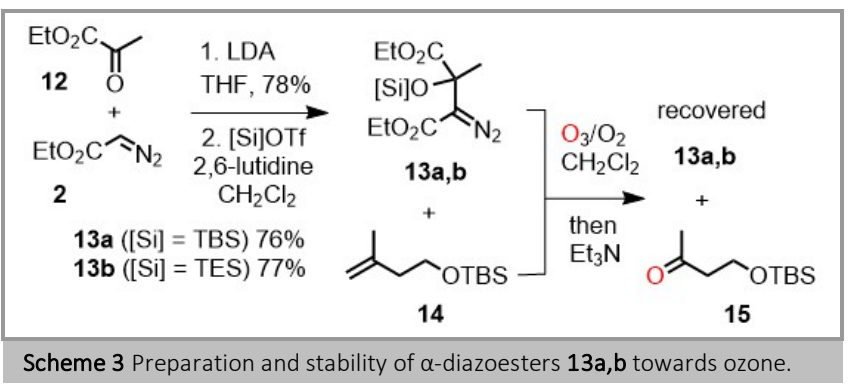

The presence of further electron-withdrawing functionality on the diazocarbonyl likely reduces the reactivity of the diazo group towards ozone. ${ }^{11}$ For example, $\alpha$-diazo- $\beta$-ketoester $16 \mathbf{a}^{12}$ (Scheme 4) was stable to ozone even at $-15^{\circ} \mathrm{C}$ for $1 \mathrm{~h}$ (alkene 14 converted to ketone 15 within $5 \mathrm{~min}$ at $-78{ }^{\circ} \mathrm{C}$ ), whereas, the diazo moiety in 13a (Scheme $3, \mathrm{CO}_{2} \mathrm{Me}$ instead of $\mathrm{CO}_{2} \mathrm{Et}$ ) is converted to the corresponding ketone on ozonolysis at $-15{ }^{\circ} \mathrm{C} .{ }^{6} \alpha$-Substituted $\alpha$-diazoesters $\mathbf{1 6 b}, \mathbf{c}$ (Scheme 4 ) were also examined, to study the effect of different substitution on the stability of the diazo moiety to ozonolysis; however, they were both reactive to ozonolysis at $-78{ }^{\circ} \mathrm{C}$, being converted to the corresponding ketones $\mathbf{1 7} \mathbf{b}, \mathbf{c}$ after addition of $\mathrm{Et}_{3} \mathrm{~N}$.

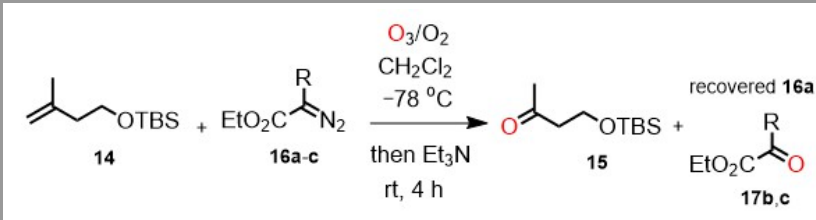

$$
\begin{aligned}
& \mathrm{R}=\underset{16 \mathbf{a}}{\mathrm{COCO}_{2} \mathrm{Et},}, \mathrm{C}_{4} \mathrm{H}_{9},
\end{aligned}
$$

Scheme 4 Examining the tolerance of $\alpha$-substituted $\alpha$-diazoesters 16 towards ozone.

Following the above observations, we focused on a synthesis of the racemic DDSQ intermediate 4 (Scheme $1, R^{\prime \prime}=O B n$ ), through diazoacetate anion addition to homoallylic $\alpha$-ketoester 20 (Scheme 5). Although many methods are known for synthesis of $\alpha$-ketoesters, 13 only a limited number of organometallic additions to oxalates are suitable for the synthesis of homoallylic $\alpha$-ketoesters. ${ }^{14}$ When the freshly prepared Grignard reagent from bromide 18a (available from the corresponding alcohol, ${ }^{2}$ see experimental) was added to a solution of dimethyl oxalate (19) in THF at $-65^{\circ} \mathrm{C}$, only a low yield of $\alpha$-ketoester 20 was obtained (11\%). Halogen-lithium exchange of iodide $18 \mathbf{b}^{2}$ using $t$-BuLi ( 2 equiv) at $-78{ }^{\circ} \mathrm{C}^{15}$ and dropwise addition of the resulting organolithium to a solution of oxalate 19 in $\mathrm{Et}_{2} \mathrm{O}\left(\right.$ at $-78{ }^{\circ} \mathrm{C}$ ) gave a similar yield $(\sim 15 \%)$. The low yield could be due to the limited solubility of dimethyl oxalate in $\mathrm{Et}_{2} \mathrm{O}$ at $-78{ }^{\circ} \mathrm{C}$, as switching from $\mathrm{Et}_{2} \mathrm{O}$ to $\mathrm{THF}$ led to an improved yield of $\alpha$-ketoester 20 (37\%). Next, diazo-alcohol 21 was prepared through the addition of lithiated ethyl diazoacetate 2 to $\alpha$-ketoester 20 (78\%).

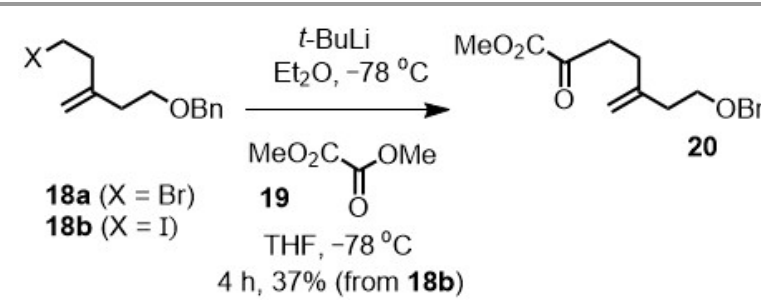

4 h, 37\% (from 18b)

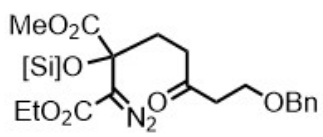

24a $([\mathrm{Si}]=$ TBS $), 58 \%$

24b $([\mathrm{Si}]=\mathrm{TMS}), 61 \%$

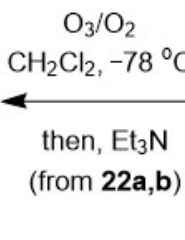<smiles>CCOC(=O)C1(O)CCC(CCOCc2ccccc2)=NC1(OC)OC</smiles>

LDA, THF

$-78{ }^{\circ} \mathrm{C}, 78 \%$

TBSOT, 6-lutidine $\mathrm{CH}_{2} \mathrm{Cl}_{2}$ (for 22a and 23a),

HMDS, imidazole, THF (for 22b and 23b)

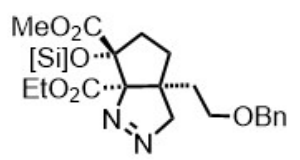

22a $([\mathrm{Si}]=\mathrm{TBS}), 18 \%$ 22b $([\mathrm{Si}]=\mathrm{TMS}), 40 \%$ 23a $([\mathrm{Si}]=\mathrm{TBS}), 37 \%$

23b $([\mathrm{Si}]=\mathrm{TMS}), 31 \%$ 
Pleasingly, and following the trend seen with the model studies (Scheme 2), no undesired intramolecular cycloaddition was observed for diazo-alcohol 21. At this stage we proceeded to protect the $\mathrm{OH}$ group, since otherwise it would be highly likely to undergo problematic $\gamma$-lactolisation with the ketone subsequently generated on ozonolysis. ${ }^{16} \mathrm{~A}$ silyl group was the protecting group of choice for this purpose. Interestingly, when diazo-alcohol $\mathbf{2 1}$ was treated with TBSOTf in the presence of 2,6-lutidine $\left(\mathrm{CH}_{2} \mathrm{Cl}_{2}, 48 \mathrm{~h}\right)$, a mixture of desired TBS ether $\mathbf{2 2 a}$ along with cycloadduct 23a (as a single diastereomer) ${ }^{17}$ was obtained (22a:23a, 1:2). Using DMAP or pyridine as the base was not successful in preventing the 1,3-dipolar cycloaddition, whereas using $\mathrm{Et}_{3} \mathrm{~N}$ led to retro-aldolisation. The partial formation of cycloadduct 23a on silylation could be rationalised by a Thorpe-Ingold effect, from the bulky silyl group. With this in mind, it was considered that minimising the steric effect by using a less-hindered silyl group might reduce the bicyclic product formation; indeed on trimethylsilylation, the ratio of silyl-ether $\mathbf{2 2 b}$ to cycloadduct $\mathbf{2 3} \mathbf{b}$ was $4: 3$ in favour of the desired non-cyclic product. Finally, ozonolysis of silyl ethers 22a and 22b led to diazoketones 24a and 24b (58\% and 61\%, respectively). The silyl ethers $\mathbf{2 2 a}, \mathbf{b}$ should be directly subjected to ozonolysis following their isolation, as they undergo intramolecular cyclisation to cycloadducts $\mathbf{2 3 a}, \mathbf{b}$ with the rate being slower for 22b (see SI: Figure S2).

In summary, we have developed a strategy for the synthesis of $\alpha$-diazo- $\varepsilon$-ketoesters, for application in squalestatin synthesis, based on chemoselective ozonolysis of unsaturated $\alpha$ diazoesters. To survive ozonolysis, $\alpha$-diazoester functionality requires to be either further substituted with a $\beta$-carbonyl group, or be sterically shielded by substituents at the $\beta$-position. Further studies will focus on construction of the enantiomerically enriched intermediate for the natural product (DDSQ).

\section{The experimental section has no title; please leave this line here}

All reactions requiring anhydrous conditions were carried out under an atmosphere of nitrogen in flame-dried glassware. Tetrahydrofuran (THF), dichloromethane and dimethylformamide (DMF) were obtained from Grubbs' drying stills. ${ }^{18}$ Flash column chromatography was carried out using silica gel (VWR chemicals, BDH), monitored by thin-layer chromatography (TLC) (Merck $60 \mathrm{~F}_{254}$ ) plates. TLC plates were viewed using ultraviolet light $\left(\lambda_{\max }=254 / 365 \mathrm{~nm}\right)$ and immersion in $\mathrm{KMnO}_{4}$, followed by heating. Except where stated otherwise, commercially available reagents were used as received. Infrared spectra were obtained using a PerkinElmer FT-IR spectrometer (Universal ATR Sampling Accessory), with absorption maxima quoted in wavenumbers $\left(\mathrm{cm}^{-1}\right)$. Peak intensities are described as broad (br), weak (w), medium (m) or strong (s). Nuclear magnetic resonance $\left({ }^{1} \mathrm{H}\right.$ NMR and ${ }^{13} \mathrm{C}$ NMR) spectra were recorded on Bruker Avance AVIIIHD 400, NEO 400, and AVIIIHD 500 spectrometers in $\mathrm{CDCl}_{3}$ (referenced to residual $\mathrm{CHCl}_{3}$ singlet at $\delta$ 7.27 for ${ }^{1} \mathrm{H}$ NMR spectra, and to the central line of $\mathrm{CDCl}_{3}$ triplet at $\delta 77.16$ for ${ }^{13} \mathrm{C}$ NMR spectra). Chemical shifts are quoted in parts per million (ppm). Coupling constants $(J)$ are measured to the nearest 0.5 Hertz $(\mathrm{Hz})$. The splittings are quoted as singlet $(\mathrm{s})$, doublet $(\mathrm{d})$, triplet $(\mathrm{t})$, quartet $(\mathrm{q})$, doublet of doublets (dd), triplet of triplets $(\mathrm{tt})$, doublet of doublet of doublets (ddd), doublet of quartets (dq), or multiplet (m). The ${ }^{13} \mathrm{C}$ signal of $\mathrm{CN}_{2}$ was often not observed due to quadrupolar relaxation. High-resolution mass spectra were obtained by electrospray ionization, using a Thermo Fisher Orbitrap Exactive mass spectrometer.

\section{Procedures}

(a) Propensity of diazoalkenes to undergo intramolecular cycloaddition

Ethyl 3a,4,5,6-tetrahydrocyclopenta[c]pyrazole-6a(3H)-carboxylate (11a)

To a solution of hydrazone $9 \mathbf{a}^{6}$ (500 $\mathrm{mg}, 1.42 \mathrm{mmol}$ ) in $\mathrm{CH}_{2} \mathrm{Cl}_{2}(2 \mathrm{ml})$ was added $\mathrm{Et}_{3} \mathrm{~N}(0.62 \mathrm{ml}, 4.4 \mathrm{mmol})$ and stirred at rt. After $3 \mathrm{~h}$, the mixture was passed through a short pad of silica $(\sim 2 \mathrm{~cm})$, and washed through with $\mathrm{CH}_{2} \mathrm{Cl}_{2}(20 \mathrm{ml})$. The eluent was evaporated under reduced pressure and the mixture purified by column chromatography (30\% $\mathrm{Et}_{2} \mathrm{O}$ in petrol) to give 1-pyrazoline 11a ( $240 \mathrm{mg}, 92 \%)$, as a yellow oil; $R_{f}=0.52$ (30\% EtOAc in petrol).

$v_{\max } / \mathrm{cm}^{-1}($ film) $296 \mathrm{w}, 2871 \mathrm{w}, 1730 \mathrm{~s}, 1556 \mathrm{w}, 1267 \mathrm{~s}, 1222 \mathrm{~s}, 1094 \mathrm{~s}$.

${ }^{1} \mathrm{H}$ NMR (500 MHz; $\left.\mathrm{CDCl}_{3}\right) \delta 4.67(1 \mathrm{H}, \mathrm{dd}, J 18.5$ and $9, \mathrm{CHCH} H \mathrm{~N}=\mathrm{N}), 4.40$ $(1 \mathrm{H}, \mathrm{dd}, J 18.5$ and $3, \mathrm{CHCHHN}=\mathrm{N}), 4.23-4.15\left(2 \mathrm{H}, \mathrm{m}, \mathrm{CH}_{2} \mathrm{CH}_{3}\right), 2.64(1 \mathrm{H}$, $\mathrm{tt}, J 9$ and $\left.3, \mathrm{CHCH}_{2} \mathrm{~N}=\mathrm{N}\right), 2.40-2.34\left(1 \mathrm{H}, \mathrm{m}, \mathrm{CHHCCO}_{2} \mathrm{Et}\right), 2.32-2.24(1 \mathrm{H}$, $\left.\mathrm{m}, \mathrm{CHHCCO}_{2} \mathrm{Et}\right), 1.85-1.76\left(1 \mathrm{H}, \mathrm{m}, \mathrm{CHCH}_{\mathrm{CHCH}}\right), 1.69-1.61(1 \mathrm{H}, \mathrm{m}$, $\left.\mathrm{CHCH}_{2} \mathrm{CH} H\right), 1.40-1.34(1 \mathrm{H}, \mathrm{m}, \mathrm{CHCHHCH} 2), 1.25\left(3 \mathrm{H}, \mathrm{t}, J\right.$ 8, $\left.\mathrm{COCH}_{2} \mathrm{CH}_{3}\right)$, 1.12-1.02 (1H, m, $\left.\mathrm{CHCH}_{2} \mathrm{CHH}\right)$.

${ }^{13} \mathrm{C}$ NMR (125 MHz; $\left.\mathrm{CDCl}_{3}\right) \delta 170.5\left(\mathrm{CO}_{2} \mathrm{Et}\right.$ ), 106.8 (quat. $\mathrm{CCO}_{2} \mathrm{Et}$ ), 86.1 $\left(\mathrm{CH}_{2} \mathrm{~N}=\mathrm{N}\right), 61.8\left(\mathrm{COCH}_{2} \mathrm{CH}_{3}\right), 39.3\left(\mathrm{CHCH}_{2} \mathrm{~N}=\mathrm{N}\right), 34.3\left(\mathrm{CH}_{2} \mathrm{CCO}_{2} \mathrm{Et}\right), 34.2$ $\left(\mathrm{CHCH}_{2} \mathrm{CH}_{2}\right), 24.0\left(\mathrm{CHCH}_{2} \mathrm{CH}_{2}\right), 14.2\left(\mathrm{COCH}_{2} \mathrm{CH}_{3}\right)$.

HRMS $m / z\left(\mathrm{M}+\mathrm{Na}^{+}\right)$found 205.0949, $\mathrm{C}_{9} \mathrm{H}_{14} \mathrm{~N}_{2}{ }^{23} \mathrm{NaO}_{2}$ requires 205.0948.

Ethyl 2-diazo-6-methylhept-6-enoate (10b) and Ethyl 3a-methyl$3 a, 4,5,6$-tetrahydrocyclo penta[c]pyrazole- $6 \mathrm{a}(3 \mathrm{H})$-carboxylate (11b)

To a solution of hydrazone $9 \mathbf{b}^{6}(45 \mathrm{mg}, 0.13 \mathrm{mmol})$ in $\mathrm{CH}_{2} \mathrm{Cl}_{2}(3 \mathrm{ml})$ was added $\mathrm{Et}_{3} \mathrm{~N}(53.0 \mu \mathrm{l}, 0.38 \mathrm{mmol})$ and stirred at rt. After $3 \mathrm{~h}$, the mixture was passed through a short pad of silica $(\sim 2 \mathrm{~cm})$, and washed through with $\mathrm{CH}_{2} \mathrm{Cl}_{2}(20 \mathrm{ml})$. The eluent was evaporated under reduced pressure and the mixture purified by column chromatography $\left(30 \% \mathrm{Et}_{2} \mathrm{O}\right.$ in petrol) to give an inseparable mixture of diazoalkene $\mathbf{1 0 b}$ and cycloadduct $\mathbf{1 1 b}$ (13 mg, 52\%, 91:9 respectively, by integration at $\mathbf{1 0 b}$ [ $\delta$ $\left.\mathrm{C}=\mathrm{CH}_{2}\right]$ and 11b $\left[\delta \mathrm{CH}_{2} \mathrm{~N}=\mathrm{N}\right]$ peaks), as a yellow oil; $R_{f}=0.54\left(30 \% \mathrm{Et}_{2} \mathrm{O}\right.$ in petrol).

$v_{\max } / \mathrm{cm}^{-1}$ (film) $2934 \mathrm{w}, 2361 \mathrm{w}, 2078 \mathrm{~s}, 1688 \mathrm{~s}, 1447 \mathrm{~m}, 1371 \mathrm{~m}, 1304$ $\mathrm{m}, 1157 \mathrm{~s}, 1110 \mathrm{~s}$.

HRMS $\left[\mathrm{M}+\mathrm{Na}^{+}\right]$found 219.1106, $\mathrm{C}_{10} \mathrm{H}_{16} \mathrm{~N}_{2}{ }^{23} \mathrm{NaO}_{2}$ requires 219.1109.

Data for diazoalkene 10b:

${ }^{1} \mathrm{H}$ NMR (500 MHz; $\left.\mathrm{CDCl}_{3}\right) \delta 4.73(1 \mathrm{H}, \mathrm{s}, \mathrm{C}=\mathrm{CHH}), 4.68(1 \mathrm{H}, \mathrm{s}, \mathrm{C}=\mathrm{CH} H)$, $4.22\left(2 \mathrm{H}, \mathrm{q}, J \mathrm{~J}, \mathrm{COCH}_{2} \mathrm{CH}_{3}\right), 2.30\left(2 \mathrm{H}, \mathrm{t}, J 7.5, \mathrm{CH}_{2} \mathrm{C}=\mathrm{CH}_{2}\right), 2.07(2 \mathrm{H}, \mathrm{t}, J 7.5$, $\left.\mathrm{CH}_{2} \mathrm{CN}=\mathrm{N}\right), 1.71\left(3 \mathrm{H}, \mathrm{s}, \mathrm{CH}_{3} \mathrm{C}=\mathrm{CH}_{2}\right), 1.66\left(2 \mathrm{H}, \mathrm{m}, \mathrm{CH}_{2} \mathrm{CH}_{2} \mathrm{CN}=\mathrm{N}\right), 1.27$ $\left(3 \mathrm{H}, \mathrm{t}, \mathrm{J} 7, \mathrm{COCH}_{2} \mathrm{CH}_{3}\right)$.

${ }^{13} \mathrm{C}$ NMR (125 MHz; $\left.\mathrm{CDCl}_{3}\right) \delta 169.7\left(\mathrm{CO}_{2} \mathrm{Et}\right), 144.9\left(\mathrm{C}=\mathrm{CH}_{2}\right), 110.7$ $\left(\mathrm{C}=\mathrm{CH}_{2}\right), 60.9\left(\mathrm{COCH}_{2} \mathrm{CH}_{3}\right), 36.9\left(\mathrm{CH}_{2} \mathrm{CN}=\mathrm{N}\right), 25.7\left(\mathrm{CH}_{2} \mathrm{CH}_{2} \mathrm{CN}=\mathrm{N}\right), 22.9$ $\left(\mathrm{CH}_{2} \mathrm{C}=\mathrm{CH}_{2}\right), 22.4\left(\mathrm{CH}_{3} \mathrm{C}=\mathrm{CH}_{2}\right), 14.7\left(\mathrm{COCH}_{2} \mathrm{CH}_{3}\right)$.

Data for cycloadduct 11b:

${ }^{1} \mathrm{H}$ NMR (500 MHz; $\left.\mathrm{CDCl}_{3}\right) \delta 4.53(0.1 \mathrm{H}, \mathrm{d}, J 18, \mathrm{CH} H \mathrm{~N}=\mathrm{N}), 4.34(0.1 \mathrm{H}, \mathrm{d}, J$ $18, \mathrm{C} H \mathrm{HN}=\mathrm{N}), 2.52-2.41(\mathrm{CH}), 1.11\left(0.3 \mathrm{H}, \mathrm{s}\right.$, quat. $\left.\mathrm{CCH}_{3}\right)$.

${ }^{13} \mathrm{C}$ NMR (125 MHz; $\left.\mathrm{CDCl}_{3}\right) \delta 167.8\left(\mathrm{CO}_{2} \mathrm{Et}\right.$ ), 105.7 (quat. $\mathrm{CCO}_{2} \mathrm{Et}$ ), 91.7 $\left(\mathrm{CH}_{2} \mathrm{~N}=\mathrm{N}\right), 61.7\left(\mathrm{COCH}_{2} \mathrm{CH}_{3}\right), 47.2$ (quat. $\left.\mathrm{CCH}_{3}\right), 42.5\left(\mathrm{CH}_{2} \mathrm{CCO}_{2} \mathrm{Et}\right), 34.0$ $\left(\mathrm{CH}_{2} \mathrm{CH}_{2} \mathrm{CCO}_{2} \mathrm{Et}\right), 23.3\left(\mathrm{CH}_{2} \mathrm{CCH}_{3}\right), 22.2$ (quat. $\left.\mathrm{CCH}_{3}\right)$.

(b) Preparation and stability of $\alpha$-substituted $\alpha$-diazoesters towards ozone

$\alpha$-Diazo- $\beta$-ketoester 16a was prepared according to a literature procedure $^{12}$ and $\alpha$-diazoester $\mathbf{1 6} \mathbf{b}$ was prepared following a procedure reported by Wang and co-workers. ${ }^{19}$ 


\section{Diethyl 3-diazo-2-hydroxy-2-methylsuccinate (25)}

The procedure of Padwa and co-workers ${ }^{5 h}$ was followed with slight modifications. A solution of LDA [prepared from $n$-BuLi (3.1 ml, 1,4 M in hexanes, $4.6 \mathrm{mmol})$ and $i$ - $\mathrm{Pr}_{2} \mathrm{NH}(600 \mu \mathrm{l}, 4.6 \mathrm{mmol})$ in THF $(5 \mathrm{ml})$ at -78 $\left.{ }^{\circ} \mathrm{C}\right]$ was added dropwise to a stirred solution of ethyl diazoacetate (2) (525 mg, $4.60 \mathrm{mmol}$ ) and ethyl pyruvate (12) (534 mg, $4.60 \mathrm{mmol}$ ) in THF $(20 \mathrm{ml})$ at $-78{ }^{\circ} \mathrm{C}$. The solution was warmed to rt and stirred for $3 \mathrm{~h}$, and then $\mathrm{AcOH}(0.26 \mathrm{ml}, 4.6 \mathrm{mmol})$ added dropwise. The mixture was extracted with $\mathrm{Et}_{2} \mathrm{O}(2 \times 15 \mathrm{ml})$, the combined organic layers washed with brine $(10 \mathrm{ml})$, dried $\left(\mathrm{MgSO}_{4}\right)$ and evaporated under reduced pressure. The residue was purified by column chromatography $(10 \%$ $\mathrm{Et}_{2} \mathrm{O}$ in petrol) to give diethyl 3-diazo-2-hydroxy-2-methylsuccinate (25) ( $825 \mathrm{mg}, 78 \%$ ), as a yellow liquid; $R_{f}=0.27$ (30\% $\mathrm{Et}_{2} \mathrm{O}$ in petrol).

$\nu_{\max } / \mathrm{cm}^{-1}$ (film) $3380 \mathrm{br}, 2956 \mathrm{~m}, 2096 \mathrm{~s}, 1745 \mathrm{~s}$ and $1698 \mathrm{~s}$.

${ }^{1} \mathrm{H}$ NMR (400 MHz; $\left.\mathrm{CDCl}_{3}\right) \delta$ 4.29-4.16 (4H, m, 2 x OCH $\left.\mathrm{H}_{2} \mathrm{Me}\right), 4.13(1 \mathrm{H}, \mathrm{s}$, $\mathrm{OH}), 1.56$ (3H, s, Me), 1.30-1.22 (6H, m, $\left.2 \times \mathrm{OCH}_{2} \mathrm{Me}\right)$.

${ }^{13} \mathrm{C}$ NMR (100 MHz; $\left.\mathrm{CDCl}_{3}\right) \delta 173.8\left(\mathrm{CO}_{2} \mathrm{Et}\right), 165.7\left(\mathrm{CO}_{2} \mathrm{Et}\right), 71.6$ (quat. C), $62.9\left(\mathrm{OCH}_{2}\right), 61.3\left(\mathrm{OCH}_{2}\right), 23.8(\mathrm{Me}), 14.5\left(\mathrm{OCH}_{2} \mathrm{CH}_{3}\right), 14.2\left(\mathrm{OCH}_{2} \mathrm{CH}_{3}\right)$.

HRMS $m / z\left(\mathrm{M}+\mathrm{Na}^{+}\right)$found: 253.0795, $\mathrm{C}_{9} \mathrm{H}_{14} \mathrm{O}_{5} \mathrm{~N}_{2}{ }^{23} \mathrm{Na}$ requires 253.0794 .

Diethyl 2-((tert-butyldimethylsilyl)oxy)-3-diazo-2-methylsuccinate (13a)

TBSOTf ( $0.4 \mathrm{ml}, 1.7 \mathrm{mmol})$ was added to 2,6-lutidine $(0.3 \mathrm{ml}, 2.6 \mathrm{mmol})$ in $\mathrm{CH}_{2} \mathrm{Cl}_{2}(1 \mathrm{ml})$ and stirred for $10 \mathrm{~min}$ at $0{ }^{\circ} \mathrm{C}$. A solution of diethyl 3diazo-2-hydroxy-2-methylsuccinate (25) (200 mg, $0.87 \mathrm{mmol}$ ) in $\mathrm{CH}_{2} \mathrm{Cl}_{2}$ $(2 \mathrm{ml})$ at $0^{\circ} \mathrm{C}$ was then added. The mixture was warmed to $\mathrm{rt}$ and stirred for $48 \mathrm{~h}$. The mixture was quenched with water $(4 \mathrm{ml})$, extracted with $\mathrm{CH}_{2} \mathrm{Cl}_{2}(3 \times 10 \mathrm{ml})$, washed with brine $(10 \mathrm{ml})$ and dried $\left(\mathrm{MgSO}_{4}\right)$. After evaporation under reduced pressure, the residue was purified by column chromatography (10\% $\mathrm{Et}_{2} \mathrm{O}$ in petrol) to give TBS ether 13a (227 mg $76 \%) ; R_{f}=0.3\left(20 \% \mathrm{Et}_{2} \mathrm{O}\right.$ in petrol).

$v_{\max } / \mathrm{cm}^{-1}$ (film) $2956 \mathrm{~m}, 2931 \mathrm{~m}, 2858 \mathrm{~m}, 2096 \mathrm{~s}, 1745 \mathrm{~s}, 1698 \mathrm{~s}, 1464 \mathrm{~m}$, 1309 s, 1255 s, 1144 s, 1069 s, 839 w.

$\left.{ }^{1} \mathrm{H} \mathrm{NMR} \mathrm{(400} \mathrm{MHz;} \mathrm{CDCl}_{3}\right) \delta 4.25-4.14\left(4 \mathrm{H}, \mathrm{m}, 2 \times \mathrm{OCH}_{2} \mathrm{CH}_{3}\right), 1.66(3 \mathrm{H}, \mathrm{s}$, $\mathrm{Me}), 1.30-1.22\left(6 \mathrm{H}, \mathrm{m}, 2 \times \mathrm{OCH}_{2} \mathrm{CH}_{3}\right), 0.86\left(9 \mathrm{H}, \mathrm{s}, \mathrm{OSiC}\left(\mathrm{CH}_{3}\right)_{3}\right), 0.11$ and $0.08\left(6 \mathrm{H}, 2 \times \mathrm{s}, \mathrm{OSi}\left(\mathrm{CH}_{3}\right)_{2}\right)$.

$\left.{ }^{13} \mathrm{C} \mathrm{NMR} \mathrm{(100} \mathrm{MHz;} \mathrm{CDCl}_{3}\right) \delta 172.0\left(\mathrm{CO}_{2} \mathrm{Et}\right), 165.0\left(\mathrm{CO}_{2} \mathrm{Et}\right), 73.7$ (quat. C), $61.9\left(\mathrm{OCH}_{2}\right), 60.9\left(\mathrm{OCH}_{2}\right), 25.9(\mathrm{Me}), 25.7\left(\mathrm{SiC}\left(\mathrm{CH}_{3}\right)_{3}\right), 18.4\left(\mathrm{SiC}\left(\mathrm{CH}_{3}\right)_{3}\right)$, $14.6\left(\mathrm{OCH}_{2} \mathrm{CH}_{3}\right), 14.2\left(\mathrm{OCH}_{2} \mathrm{CH}_{3}\right)-3.0\left(\mathrm{OSi}\left(\mathrm{CH}_{3}\right)_{2}\right),-3.5\left(\mathrm{OSi}\left(\mathrm{CH}_{3}\right)_{2}\right)$.

HRMS [M+Na+] found: 367.1656. $\mathrm{C}_{15} \mathrm{H}_{28} \mathrm{O}_{5} \mathrm{~N}_{2}{ }^{28} \mathrm{Si}^{23} \mathrm{Na}$ requires 367.1659.

\section{Diethyl 3-diazo-2-methyl-2-((triethylsilyl)oxy)succinate (13b)}

Following the procedure for silyl ether 13a above, but using TESOTf, diethyl 3-diazo-2-hydroxy-2-methylsuccinate (25) (200 mg, $0.87 \mathrm{mmol}$ ) gave after column chromatography (10\% $\mathrm{Et}_{2} \mathrm{O}$ in petrol) TES ether $\mathbf{1 3 b}$ (230 mg, 77\%), as a yellow oil; $R_{f}=0.3\left(20 \% \mathrm{Et}_{2} \mathrm{O}\right.$ in petrol).

$v_{\max } / \mathrm{cm}^{-1}($ film) $2956 \mathrm{~m}, 2931 \mathrm{~m}, 2858 \mathrm{~m}, 2096 \mathrm{~s}, 1745 \mathrm{~s}, 1698 \mathrm{~s}, 1464 \mathrm{~m}$, 1309 s, 1255 s, 1144 s, 1069 s, 839 w.

${ }^{1} \mathrm{H}$ NMR (400 MHz; $\left.\mathrm{CDCl}_{3}\right) \delta$ 4.25-4.13 (4H, m, 2 x OCH $\left.\mathrm{OCH}_{3}\right), 1.67(3 \mathrm{H}, \mathrm{s}$, $\mathrm{Me}), 1.27\left(3 \mathrm{H}, \mathrm{t}, J 7, \mathrm{OCH}_{2} \mathrm{CH}_{3}\right), 1.24\left(3 \mathrm{H}, \mathrm{t}, J 7, \mathrm{OCH}_{2} \mathrm{CH}_{3}\right), 0.93(9 \mathrm{H}, \mathrm{t}, J$, $\left.\mathrm{OSi}\left(\mathrm{CH}_{2} \mathrm{CH}_{3}\right)_{3}\right), 0.62\left(6 \mathrm{H}, \mathrm{q}, J=8, \mathrm{OSi}\left(\mathrm{CH}_{2} \mathrm{CH}_{3}\right)_{3}\right)$.

${ }^{13} \mathrm{C}$ NMR (100 MHz; $\left.\mathrm{CDCl}_{3}\right) \delta 172.0\left(\mathrm{CO}_{2} \mathrm{Et}\right), 165.1\left(\mathrm{CO}_{2} \mathrm{Et}\right), 73.6$ (quat. C), $61.9\left(\mathrm{OCH}_{2}\right), 60.9\left(\mathrm{OCH}_{2}\right), 25.9(\mathrm{Me}), 14.6\left(\mathrm{OCH}_{2} \mathrm{CH}_{3}\right), 14.2\left(\mathrm{OCH}_{2} \mathrm{CH}_{3}\right) 6.9$ $\left(\mathrm{OSi}\left(\mathrm{CH}_{2} \mathrm{CH}_{3}\right)_{3}\right), 6.1\left(\mathrm{OSi}\left(\mathrm{CH}_{2} \mathrm{CH}_{3}\right)_{3}\right)$.

HRMS [M+Na+ $a^{+}$found: 367.1656. $\mathrm{C}_{15} \mathrm{H}_{28} \mathrm{O}_{5} \mathrm{~N}_{2}{ }^{23} \mathrm{Na}^{28} \mathrm{Si}$ requires 367.1659.

\section{Ethyl 2-diazo-2-(1-((trimethylsilyl)oxy)cyclopentyl)acetate (16c)}

TMSCl (127 $\mu \mathrm{l}, 1.0 \mathrm{mmol})$ was added slowly to a solution of ethyl 2diazo-2-(1-hydroxycyclopentyl)acetate $\mathrm{e}^{5 \mathrm{~h}}(100 \mathrm{mg}, 0.50 \mathrm{mmol})$ and imidazole (170 mg, $2.50 \mathrm{mmol})$ in DMF $(1.4 \mathrm{ml})$ at $0{ }^{\circ} \mathrm{C}$. After $4 \mathrm{~h}$ at rt,
$\mathrm{Et}_{2} \mathrm{O}(5 \mathrm{ml})$ was added, the organic layer was washed with water $(2 \times 2$ $\mathrm{ml})$, brine $(2 \mathrm{ml})$ and dried $\left(\mathrm{Na}_{2} \mathrm{SO}_{4}\right)$. After evaporation under reduced pressure, the residue was purified by column chromatography $(0-10 \%$ $\mathrm{Et}_{2} \mathrm{O}$ in petrol) to give silyl ether $\mathbf{1 6 c}$ (118 $\left.\mathrm{mg}, 87 \%\right)$, as a yellow liquid; $R_{f}=0.59$ (10\% $\mathrm{Et}_{2} \mathrm{O}$ in petrol).

$v_{\max } / \mathrm{cm}^{-1}($ film) $2960 \mathrm{~m}, 1714 \mathrm{~s}, 1370 \mathrm{w}, 1204 \mathrm{~m}, 1040 \mathrm{~m}$.

${ }^{1} \mathrm{H}$ NMR (400 MHz; $\left.\mathrm{CDCl}_{3}\right) \delta 4.22\left(2 \mathrm{H}, \mathrm{q}, J\right.$ 7, $\left.\mathrm{OCH}_{2} \mathrm{CH}_{3}\right), 2.05-1.99(2 \mathrm{H}, \mathrm{m}$, $\left.\mathrm{CH}_{2}\right), 1.94-1.86\left(2 \mathrm{H}, \mathrm{m}, \mathrm{CH}_{2}\right), 1.84-1.78\left(2 \mathrm{H}, \mathrm{m}, \mathrm{CH}_{2}\right), 1.70-1.63(2 \mathrm{H}, \mathrm{m}$, $\left.\mathrm{CH}_{2}\right), 1.28\left(3 \mathrm{H}, \mathrm{t}, J \mathrm{~J} .0, \mathrm{OCH}_{2} \mathrm{CH}_{3}\right), 1.10$ (OTMS).

${ }^{13} \mathrm{C}$ NMR (100 MHz; $\left.\mathrm{CDCl}_{3}\right) \delta 165.7$ ( $\left.\mathrm{CO}_{2} \mathrm{Et}\right), 81.0$ (COTMS), 60.6 $\left(\mathrm{OCH}_{2} \mathrm{CH}_{3}\right), 40.1\left(2 \mathrm{x} \mathrm{CH}_{2}\right), 22.8\left(2 \times \mathrm{CH}_{2}\right), 14.7\left(\mathrm{OCH}_{2} \mathrm{CH}_{3}\right), 1.5$ $\left(\mathrm{OSi}\left(\mathrm{CH}_{3}\right)_{3}\right)$.

HRMS $m / z \quad\left(M+\mathrm{Na}^{+}\right)$found: 293.1292. $\mathrm{C}_{12} \mathrm{H}_{22} \mathrm{O}_{3} \mathrm{~N}_{2}{ }^{23} \mathrm{Na}^{28} \mathrm{Si}$ requires 293.1291.

\section{General procedure for ozonolysis}

A stream of $\mathrm{O}_{3} / \mathrm{O}_{2}$ was bubbled through a mixture of $\alpha$-diazoester (1 mmol) and alkene $\mathbf{1 4}^{20}(1 \mathrm{mmol})$ in $\mathrm{CH}_{2} \mathrm{Cl}_{2}$ at $-78{ }^{\circ} \mathrm{C}$ until the colour of the reaction mixture changed from yellow to blue $(\sim 5 \mathrm{~min})$. Then the excess of $\mathrm{O}_{3}$ was removed by bubbling $\mathrm{N}_{2}$ through the reaction mixture, followed by addition of $\mathrm{Et}_{3} \mathrm{~N}$ ( $\left.3 \mathrm{mmol}\right)$. After $4 \mathrm{~h}$ at $\mathrm{rt}$, the reaction mixture was evaporated under reduced pressure and the residue analysed by ${ }^{1} \mathrm{H}$ NMR (see SI: Figure S1).

\section{Ethyl 2-oxohexanoate (17b)}

Following the general ozonolysis procedure above, $\alpha$-diazoester $\mathbf{1 6 b}$ (100 mg, $0.58 \mathrm{mmol}$ ) was oxidised to give after column chromatography (5\% EtOAc in petrol) an inseparable mixture of $\alpha$-ketoester $\mathbf{1 7} \mathbf{b}$ and the corresponding trioxane trimer $\mathbf{2 6}(66 \mathrm{mg}, 73 \%, \sim 60: 40$ respectively, by integration at 17b $\left[\delta \mathrm{CH}_{2} \mathrm{CH}_{2} \mathrm{CO}\right]$ and $26\left[\delta \mathrm{CH}_{2} \mathrm{CH}_{2} \mathrm{CO}\right]$ peaks), as a colourless liquid; $R_{f}=0.56$ ( $5 \%$ EtOAc in petrol).

$v_{\max } / \mathrm{cm}^{-1}$ (film) $2961 \mathrm{~m}, 2935 \mathrm{~m}, 1750 \mathrm{~s}, 1727 \mathrm{~s}, 1466 \mathrm{~s}, 1275 \mathrm{~m}, 1246 \mathrm{~m}$, $1142 \mathrm{~m}, 1120 \mathrm{~m}, 1048 \mathrm{~s}, 856 \mathrm{~m}$.

Data for $\alpha$-ketoester $\mathbf{1 7 b}$ :

$\left.{ }^{1} \mathrm{H} \mathrm{NMR} \mathrm{(400} \mathrm{MHz;} \mathrm{CDCl}_{3}\right) \delta 4.30\left(2 \mathrm{H}, \mathrm{q}, J\right.$ 7, $\left.\mathrm{OCH}_{2} \mathrm{CH}_{3}\right), 2.81(2 \mathrm{H}, \mathrm{t}, J$, $\mathrm{CH}_{2} \mathrm{CH}_{2} \mathrm{CO}$ ), 1.68-1.55 (2H, m, $\left.\mathrm{CH}_{2} \mathrm{CH}_{2} \mathrm{CO}\right), 1.42-1.27\left(5 \mathrm{H}, \mathrm{m}, \mathrm{CH}_{2}\right.$ and $\left.\mathrm{CO}_{2} \mathrm{CH}_{2} \mathrm{CH}_{3}\right), 0.91\left(3 \mathrm{H}, \mathrm{t}, J 7, \mathrm{OCH}_{2} \mathrm{CH}_{3}\right)$.

${ }^{13} \mathrm{C} \quad \mathrm{NMR}\left(100 \mathrm{MHz} ; \mathrm{CDCl}_{3}\right) \quad \delta \quad 194.9 \quad(\mathrm{C}=0), 161.5 \quad\left(\mathrm{CO}_{2} \mathrm{Et}\right), 62.5$ $\left(\mathrm{CO}_{2} \mathrm{CH}_{2} \mathrm{CH}_{3}\right), 39.1\left(\mathrm{CH}_{2} \mathrm{CO}\right), 25.2\left(\mathrm{CH}_{2} \mathrm{CH}_{2} \mathrm{CO}\right), 22.2\left(\mathrm{CH}_{3} \mathrm{CH}_{2} \mathrm{CH}_{2}\right), 14.1$ $\left(\mathrm{OCH}_{2} \mathrm{CH}_{3}\right), 13.9\left(\mathrm{CH}_{2} \mathrm{CH}_{3}\right)$.

HRMS $m / z\left(\mathrm{M}+\mathrm{Na}^{+}\right)$found: $181.0836 . \mathrm{C}_{8} \mathrm{H}_{14} \mathrm{O}_{3}{ }^{23} \mathrm{Na}$ requires 181.0835 .

Data for triethyl 2,4,6-tributyl-1,3,5-trioxane-2,4,6-tricarboxylate 26:

${ }^{1} \mathrm{H} \mathrm{NMR}\left(400 \mathrm{MHz} ; \mathrm{CDCl}_{3}\right.$ ) $\delta 4.27-4.21\left(1 \mathrm{H}, \mathrm{m}, \mathrm{OCH}_{2} \mathrm{CH}_{3}\right), 2.10-1.91(1 \mathrm{H}$, m, $\left.\mathrm{CH}_{2} \mathrm{CH}_{2} \mathrm{CO}\right), 1.42-1.27\left(5 \mathrm{H}, \mathrm{m}, 2 \times \mathrm{CH}_{2}\right.$ and $\left.\mathrm{CO}_{2} \mathrm{CH}_{2} \mathrm{CH}_{3}\right), 0.88(2 \mathrm{H}$, $\mathrm{t}, \mathrm{OCH}_{2} \mathrm{CH}_{3}$ ).

${ }^{13} \mathrm{C}$ NMR (100 MHz; $\left.\mathrm{CDCl}_{3}\right) \delta 168.2\left(\mathrm{CO}_{2} \mathrm{Et}\right), 107.6$ (quat. $\left.\mathrm{C}\right), 62.1$ $\left(\mathrm{CO}_{2} \mathrm{CH}_{2} \mathrm{CH}_{3}\right), 31.6\left(\mathrm{CH}_{2} \mathrm{CO}\right), 25.0\left(\mathrm{CH}_{2} \mathrm{CH}_{2} \mathrm{CO}\right), 22.6\left(\mathrm{CH}_{3} \mathrm{CH}_{2} \mathrm{CH}_{2}\right), 14.2$ $\left(\mathrm{OCH}_{2} \mathrm{CH}_{3}\right), 13.8\left(\mathrm{CH}_{2} \mathrm{CH}_{3}\right)$.

\section{Ethyl 2-oxo-2-(1-((trimethylsilyl)oxy)cyclopentyl)acetate (17c)}

Following the general ozonolysis procedure above, TMS ether 16c (47 $\mathrm{mg}, 0.17 \mathrm{mmol}$ ) was oxidised to give after column chromatography $(10 \%$ $\mathrm{Et}_{2} \mathrm{O}$ in petrol) $\alpha$-ketoester 17c (17 mg, 39\%), as a colourless liquid; $R_{f}=$ 0.5 (10\% $\mathrm{Et}_{2} \mathrm{O}$ in petrol).

$v_{\max } / \mathrm{cm}^{-1}$ (film) $2958 \mathrm{~m}, 1727$ s, 1251 s, 1216 m, 1066 s, 1028 m, 917 m, $839 \mathrm{~s}$.

${ }^{1} \mathrm{H}$ NMR (400 MHz; $\left.\mathrm{CDCl}_{3}\right) \delta 4.31\left(2 \mathrm{H}, \mathrm{q}, J\right.$ 7, $\left.\mathrm{OCH}_{2} \mathrm{CH}_{3}\right), 2.26-2.15(2 \mathrm{H}, \mathrm{m}$, $\left.\mathrm{CH}_{2}\right), 1.93-1.87\left(2 \mathrm{H}, \mathrm{m}, \mathrm{CH}_{2}\right), 1.85-1.78\left(2 \mathrm{H}, \mathrm{m}, \mathrm{CH}_{2}\right), 1.77-1.69(2 \mathrm{H}, \mathrm{m}$, $\left.\mathrm{CH}_{2}\right), 1.36\left(3 \mathrm{H}, \mathrm{t}, J \mathrm{~J}, \mathrm{OCH}_{2} \mathrm{CH}_{3}\right), 0.13$ (OTMS). 
${ }^{13} \mathrm{C}$ NMR (100 MHz; $\left.\mathrm{CDCl}_{3}\right) \delta 200.0$ (C=0), $165.2\left(\mathrm{CO}_{2} \mathrm{Et}\right.$ ), 88.8 (COTMS), $61.9\left(\mathrm{OCH}_{2} \mathrm{CH}_{3}\right), 39.0\left(2 \times \mathrm{CH}_{2}\right), 24.7\left(2 \times \mathrm{CH}_{2}\right), 14.2\left(\mathrm{OCH}_{2} \mathrm{CH}_{3}\right), 1.8$ $\left(\mathrm{OSi}\left(\mathrm{CH}_{3}\right)_{3}\right)$.

HRMS $m / z \quad\left(\mathrm{M}+\mathrm{Na}^{+}\right)$found: 281.1180. $\mathrm{C}_{12} \mathrm{H}_{22} \mathrm{O}_{4}{ }^{23} \mathrm{Na}^{28} \mathrm{Si}$ requires 281.1179 .

\section{(c) Synthesis of $\alpha$-diazo- $\varepsilon$-ketoesters $\mathbf{2 4 a}, \mathbf{b}$.}

\section{(((5-Bromo-3-methylenepentyl)oxy)methyl)benzene (18a)}

$\mathrm{Ph}_{3} \mathrm{P}$ (365 mg, $1.39 \mathrm{mmol}$ ) and $\mathrm{CBr}_{4}(577 \mathrm{mg}, 1.74 \mathrm{mmol})$ was added to a solution of 5-(benzyloxy)-3-methylenepentan-1-ol ${ }^{2}$ (240 mg, $1.16 \mathrm{mmol}$ ) in $\mathrm{CH}_{2} \mathrm{Cl}_{2}(8 \mathrm{ml})$ at $\mathrm{rt}$. After $10 \mathrm{~min}$, the reaction mixture was poured into sat. aq. $\mathrm{NaHCO}_{3}(5 \mathrm{ml})$ and the aq. layer was extracted with $\mathrm{CH}_{2} \mathrm{Cl}_{2}(2 \times 5$ $\mathrm{ml})$. The combined organic layers were washed with brine $(5 \mathrm{ml})$, dried $\left(\mathrm{MgSO}_{4}\right)$, concentrated under reduced pressure and purified by column chromatography (5\% $\mathrm{Et}_{2} \mathrm{O}$ in petrol) to give bromide 19a $(225 \mathrm{mg}$, $72 \%$ ), as a colourless oil; $R_{f}=0.41$ ( $5 \% \mathrm{Et}_{2} \mathrm{O}$ in petrol).

$v_{\max } / \mathrm{cm}^{-1}($ film) $2858 \mathrm{w}, 1646 \mathrm{~m}, 1453 \mathrm{~m}, 1208 \mathrm{~m}, 1098 \mathrm{~s}, 901 \mathrm{~s}, 736 \mathrm{~s}$, $697 \mathrm{~s}$.

${ }^{1} \mathrm{H}$ NMR (400 MHz; $\mathrm{CDCl}_{3}$ ) $\delta$ 7.40-7.25 (5H, m, $\mathrm{Ar} H$ ), $4.94(1 \mathrm{H}, \mathrm{s}, \mathrm{C}=\mathrm{CH} H$ ), $4.89(1 \mathrm{H}, \mathrm{s}, \mathrm{C}=\mathrm{CHH}), 4.53\left(2 \mathrm{H}, \mathrm{s}, \mathrm{CH}_{2} \mathrm{Ph}\right), 3.59\left(2 \mathrm{H}, \mathrm{t}, J \mathrm{~J}, \mathrm{CH}_{2} \mathrm{OBn}\right), 3.47$ $\left(2 \mathrm{H}, \mathrm{t}, J \mathrm{~T}, \mathrm{CH}_{2} \mathrm{Br}\right), 2.62\left(2 \mathrm{H}, \mathrm{t}, J 7, \mathrm{CH}_{2} \mathrm{CH}_{2} \mathrm{Br}\right), 2.36(2 \mathrm{H}, \mathrm{t}, J$ 7.0, $\mathrm{CH}_{2} \mathrm{CH}_{2} \mathrm{OBn}$ ).

${ }^{13} \mathrm{C}$ NMR (100 MHz; $\left.\mathrm{CDCl}_{3}\right) \delta 143.7\left(C=\mathrm{CH}_{2}\right), 138.4(\mathrm{ArC}), 128.5(\mathrm{ArCH})$, $127.8(\mathrm{ArCH}), \quad 127.7(\mathrm{ArCH}), \quad 113.4\left(\mathrm{C}=\mathrm{CH}_{2}\right), \quad 73.1 \quad\left(\mathrm{CH}_{2} \mathrm{Ph}\right), \quad 68.9$ $\left(\mathrm{CH}_{2} \mathrm{OBn}\right), 39.7\left(\mathrm{CH}_{2} \mathrm{CH}_{2} \mathrm{Br}\right), 35.9\left(\mathrm{CH}_{2} \mathrm{CH}_{2} \mathrm{OBn}\right), 31.0\left(\mathrm{CH}_{2} \mathrm{Br}\right)$.

HRMS $m / z \quad\left(\mathrm{M}+\mathrm{Na}^{+}\right)$found: 291.0354. $\mathrm{C}_{13} \mathrm{H}_{17} \mathrm{O}^{79} \mathrm{Br}^{23} \mathrm{Na}$ requires 291.0355.

\section{Methyl 7-(benzyloxy)-5-methylene-2-oxoheptanoate (20)}

To a solution of (((5-iodo-3-methylenepentyl)oxy)methyl)benzene $(\mathbf{1 8 b})^{2}(100 \mathrm{mg}, 0.31 \mathrm{mmol})$ in $\mathrm{Et}_{2} \mathrm{O}(3 \mathrm{ml})$ at $-78{ }^{\circ} \mathrm{C}$ was added $t$-BuLi $(0.44 \mathrm{ml}, 1.7 \mathrm{M}$ in pentane, $0.75 \mathrm{mmol})$ dropwise. After $1 \mathrm{~h}$, the reaction mixture was transferred slowly by cannula to a solution of dimethyl oxalate (19) (186 mg, $1.58 \mathrm{mmol})$ in THF $(0.5 \mathrm{ml})$ at $-78{ }^{\circ} \mathrm{C}$ and stirred for $4 \mathrm{~h}$. Then, sat. aq. $\mathrm{NH}_{4} \mathrm{Cl}(2 \mathrm{ml})$ was added and the mixture allowed to warm to rt. The aq. layer was extracted with $\mathrm{Et}_{2} \mathrm{O}(2 \times 5 \mathrm{ml})$ and the combined organic layers were washed with water $(5 \mathrm{ml})$, brine $(5 \mathrm{ml})$, dried $\left(\mathrm{MgSO}_{4}\right)$, and concentrated under reduced pressure. Purification of the residue by column chromatography $\left(20 \% \mathrm{Et}_{2} \mathrm{O}\right.$ in petrol) gave $\alpha$ ketoester 20 (32 mg, 37\%), as a colourless liquid; $R_{f}=0.15\left(20 \% \mathrm{Et}_{2} \mathrm{O}\right.$ in petrol).

$v_{\max } / \mathrm{cm}^{-1}$ (film) $2931 \mathrm{~m}, 1743 \mathrm{~s}, 1452 \mathrm{w}, 1276 \mathrm{~m}, 1099 \mathrm{~m}, 716 \mathrm{w}$.

${ }^{1} \mathrm{H}$ NMR (500 MHz; $\left.\mathrm{CDCl}_{3}\right) \delta$ 7.40-7.26 (5H, m, $\left.\mathrm{Ar} H\right), 4.84(1 \mathrm{H}, \mathrm{s}, \mathrm{C}=\mathrm{CH} H)$, $4.80(1 \mathrm{H}, \mathrm{s}, \mathrm{C}=\mathrm{CHH}), 4.51\left(2 \mathrm{H}, \mathrm{s}, \mathrm{CH}_{2} \mathrm{Ph}\right), 3.86\left(3 \mathrm{H}, \mathrm{s}, \mathrm{CO}_{2} \mathrm{Me}\right), 3.58(2 \mathrm{H}, \mathrm{t}$, $\left.J \quad 7, \mathrm{CH}_{2} \mathrm{OBn}\right), 3.00\left(2 \mathrm{H}, \mathrm{t}, J \quad 7, \mathrm{CH}_{2} \mathrm{C}=0\right), 2.42-2.33(4 \mathrm{H}, \mathrm{m}$, $\left.\mathrm{CH}_{2} \mathrm{C}\left(=\mathrm{CH}_{2}\right) \mathrm{CH}_{2}\right)$.

${ }^{13} \mathrm{C} \mathrm{NMR}\left(125 \mathrm{MHz} ; \mathrm{CDCl}_{3}\right) \delta 193.6(\mathrm{C}=0), 161.5\left(\mathrm{CO}_{2} \mathrm{Me}\right), 144.7\left(\mathrm{C}=\mathrm{CH}_{2}\right)$, 138.4 ( $\mathrm{ArC}), 128.5(\mathrm{ArCH}), 127.8(\mathrm{ArCH}), 127.7(\mathrm{ArCH}), 111.5\left(\mathrm{C}=\mathrm{CH}_{2}\right)$, $73.1\left(\mathrm{CH}_{2} \mathrm{Ph}\right), 68.9\left(\mathrm{CH}_{2} \mathrm{OBn}\right), 53.1\left(\mathrm{CO}_{2} \mathrm{Me}\right), 37.7\left(\mathrm{CH}_{2} \mathrm{CH}_{2} \mathrm{OBn}\right), 36.5$ $\left(\mathrm{CH}_{2} \mathrm{CH}_{2} \mathrm{C}=0\right), 29.3\left(\mathrm{CH}_{2} \mathrm{CH}_{2} \mathrm{C}=0\right)$.

HRMS $m / z\left(\mathrm{M}+\mathrm{Na}^{+}\right)$found: 299.1254. $\mathrm{C}_{16} \mathrm{H}_{20} \mathrm{O}_{4}{ }^{23} \mathrm{Na}$ requires 299.1264 .

4-Ethyl 1-methyl 2-(5-(benzyloxy)-3-methylenepentyl)-3-diazo-2hydroxysuccinate (21)

A solution of LDA [prepared from $n$-BuLi $(90 \mu \mathrm{l}, 2.5 \mathrm{M}$ in hexanes, 0.22 $\mathrm{mmol})$ and $i-\operatorname{Pr}_{2} \mathrm{NH}(30 \mu \mathrm{l}, 0.22 \mathrm{mmol})$ in $\mathrm{THF}(1 \mathrm{ml})$ at $\left.-78{ }^{\circ} \mathrm{C}\right]$ was added dropwise to a solution of ethyl diazoacetate (2) (23 $\mu \mathrm{l}, 0.22 \mathrm{mmol})$ and $\alpha$-ketoester 20 (36 mg, $0.13 \mathrm{mmol})$ in THF $(350 \mu \mathrm{l})$ at $-78^{\circ} \mathrm{C}$. After 2 $h$, the reaction mixture was quenched with $\mathrm{NH}_{4} \mathrm{Cl}(2 \mathrm{ml})$, extracted with $\mathrm{Et}_{2} \mathrm{O}(2 \times 5 \mathrm{ml})$, and dried $\left(\mathrm{MgSO}_{4}\right)$. After evaporation under reduced pressure, the residue was purified by a short column chromatography (10\% EtOAc in petrol) to give diazo-alcohol 21 (40 mg, 78\%), as a yellow oil; $R_{f}=0.25$ ( $50 \% \mathrm{Et}_{2} \mathrm{O}$ in petrol). $v_{\max } / \mathrm{cm}^{-1}$ (film) 3390 br, 3027 w, 2920 m, 1732 s, 1453 m, 1261 s, 1097 s, $739 \mathrm{~m}, 699 \mathrm{~m}$.

${ }^{1} \mathrm{H}$ NMR (500 MHz; $\left.\mathrm{CDCl}_{3}\right) \delta$ 7.39-7.26 (5H, m, ArH), 4.81 (2H, d, J 11.5, $\left.\mathrm{C}=\mathrm{CH}_{2}\right), 4.50\left(2 \mathrm{H}, \mathrm{s}, \mathrm{CH} \mathrm{H}_{2} \mathrm{Ph}\right), 4.28\left(2 \mathrm{H}, \mathrm{m}, \mathrm{CO}_{2} \mathrm{CH}_{2} \mathrm{CH}_{3}\right), 3.78(3 \mathrm{H}, \mathrm{s}$, $\left.\mathrm{CO}_{2} \mathrm{Me}\right), 3.56\left(2 \mathrm{H}, \mathrm{t}, J 7, \mathrm{CH}_{2} \mathrm{OBn}\right), 2.34\left(2 \mathrm{H}, \mathrm{t}, J 7, \mathrm{CH}_{2} \mathrm{CH}_{2} \mathrm{OBn}\right), 2.32-2.26$ $(1 \mathrm{H}, \mathrm{m}, \mathrm{CH} H \mathrm{C}(\mathrm{OH})), 2.02-1.97(1 \mathrm{H}, \mathrm{m}, \mathrm{CH} \mathrm{HC}(\mathrm{OH})), 1.96-1.90(2 \mathrm{H}, \mathrm{m}$, $\left.\mathrm{CH}_{2} \mathrm{CH}_{2} \mathrm{C}(\mathrm{OH})\right), 1.27\left(3 \mathrm{H}, \mathrm{t}, J 7, \mathrm{CO}_{2} \mathrm{CH}_{2} \mathrm{CH}_{3}\right)$.

${ }^{13} \mathrm{C}$ NMR (125 MHz; $\left.\mathrm{CDCl}_{3}\right) \delta 173.6\left(\mathrm{CO}_{2} \mathrm{Me}\right), 165.9\left(\mathrm{CO}_{2} \mathrm{Et}\right), 145.2$ $\left(C=\mathrm{CH}_{2}\right), 138.5(\mathrm{ArC}), 128.5(\mathrm{ArCH}), 127.8(\mathrm{ArCH}), 127.7(\mathrm{ArCH}), 111.5$ $\left(\mathrm{C}=\mathrm{CH}_{2}\right), 73.8$ (quat. $\left.\mathrm{C}(\mathrm{OH}) \mathrm{CO}_{2} \mathrm{Me}\right), 73.1\left(\mathrm{CH}_{2} \mathrm{Ph}\right), 68.9\left(\mathrm{CH}_{2} \mathrm{OBn}\right), 61.4$ $\left(\mathrm{CO}_{2} \mathrm{CH}_{2} \mathrm{CH}_{3}\right), 53.6\left(\mathrm{CO}_{2} \mathrm{Me}\right), 36.4$ and $34.6\left(\mathrm{CH}_{2} \mathrm{C}\left(=\mathrm{CH}_{2}\right) \mathrm{CH}_{2}\right), 29.7$ $\left(\mathrm{CH}_{2} \mathrm{C}(\mathrm{OH})\right), 14.5\left(\mathrm{CO}_{2} \mathrm{CH}_{2} \mathrm{CH}_{3}\right)$.

HRMS $m / z\left(\mathrm{M}+\mathrm{H}^{+}\right)$found: $391.1882 . \mathrm{C}_{20} \mathrm{H}_{27} \mathrm{O}_{6} \mathrm{~N}_{2}$ requires 391.1863.

4-Ethyl 1-methyl 2-(5-(benzyloxy)-3-methylenepentyl)-2-((tertbutyldimethylsilyl)oxy)-3-diazosuccinate (22a) and 6a-Ethyl 6methyl (3aR, 6S, 6aR)-3a-(2-(benzyloxy)ethyl)-6-((tert-butyl dimethylsilyl)oxy)-3a,4,5,6-tetrahydrocyclopenta[c]pyrazole-6,6a (3H)-dicarboxylate (23a)

TBSOTf ( $35 \mu \mathrm{l}, 0.15 \mathrm{mmol})$ was added to a solution of 2,6-lutidine ( $26 \mu \mathrm{L}$, $0.22 \mathrm{mmol})$ in $\mathrm{CH}_{2} \mathrm{Cl}_{2}(200 \mu \mathrm{l})$ at $0{ }^{\circ} \mathrm{C}$ and stirred for $15 \mathrm{~min}$. A solution of diazo-alcohol 21 ( $30 \mathrm{mg}, 0.076 \mathrm{mmol})$ in $\mathrm{CH}_{2} \mathrm{Cl}_{2}(200 \mu \mathrm{l})$ was added. The reaction mixture was stirred for $48 \mathrm{~h}$ at $\mathrm{rt}$, then water $(1 \mathrm{ml})$ was added, extracted with $\mathrm{CH}_{2} \mathrm{Cl}_{2}(2 \times 5 \mathrm{ml})$, dried $\left(\mathrm{MgSO}_{4}\right)$ and evaporated under reduced pressure. The crude mixture was purified by column chromatography (0-20\% $\mathrm{Et}_{2} \mathrm{O}$ in petrol).

First eluted TBS ether 22a (7 mg, 18\%), as a yellow oil; $R_{f}=0.23(20 \%$ $\mathrm{Et}_{2} \mathrm{O}$ in petrol).

$v_{\max } / \mathrm{cm}^{-1}$ (film) $2953 \mathrm{~m}, 2857 \mathrm{~m}, 2096 \mathrm{~s}, 1745 \mathrm{~s}, 1701 \mathrm{~s}, 1454 \mathrm{w}, 1369$ m, 1304 s, $1254 \mathrm{~m}, 1135 \mathrm{~s}, 838 \mathrm{~s}, 780 \mathrm{~m}$.

${ }^{1} \mathrm{H}$ NMR (400 MHz; $\left.\mathrm{CDCl}_{3}\right) \delta$ 7.37-7.26 (5H, m, $\left.\mathrm{Ar} H\right), 4.80\left(2 \mathrm{H}, \mathrm{s}, \mathrm{C}=\mathrm{CH}_{2}\right)$, $4.50\left(2 \mathrm{H}, \mathrm{s}, \mathrm{CH}_{2} \mathrm{Ph}\right), 4.26-4.11\left(2 \mathrm{H}, \mathrm{m}, \mathrm{CO}_{2} \mathrm{CH}_{2} \mathrm{CH}_{3}\right), 3.72\left(3 \mathrm{H}, \mathrm{s}, \mathrm{CO}_{2} \mathrm{Me}\right)$, $3.55\left(2 \mathrm{H}, \mathrm{t}, J \mathrm{~J}, \mathrm{CH} \mathrm{OBBn}_{2}, 2.33\left(2 \mathrm{H}, \mathrm{t}, J \mathrm{~J}, \mathrm{CH}_{2} \mathrm{CH}_{2} \mathrm{OBn}\right), 2.24-1.94(4 \mathrm{H}, \mathrm{m}\right.$, $\mathrm{CH}_{2} \mathrm{CH}_{2} \mathrm{C}(\mathrm{OTBS})$ ), 1.24 (3H, t, J 7, $\left.\mathrm{CO}_{2} \mathrm{CH}_{2} \mathrm{CH}_{3}\right), 0.88$ (9H, s, OSiCMes), 0.11 and $0.05\left(6 \mathrm{H}, 2 \times \mathrm{s}, \mathrm{OSi}\left(\mathrm{CH}_{3}\right)_{2}\right)$.

${ }^{13} \mathrm{C}$ NMR (100 MHz; $\left.\mathrm{CDCl}_{3}\right) \delta 173.6\left(\mathrm{CO}_{2} \mathrm{Me}\right), 145.6\left(C=\mathrm{CH}_{2}\right), 138.5(\mathrm{ArC})$, $128.5(\mathrm{ArCH}), 127.8(\mathrm{ArCH}), 127.7(\mathrm{ArCH}), 111.2\left(\mathrm{C}=\mathrm{CH}_{2}\right), 76.0$ (quat. $C$ (OTBS) $\left.\mathrm{CO}_{2} \mathrm{Me}\right), 73.1\left(\mathrm{CH}_{2} \mathrm{Ph}\right), 68.9\left(\mathrm{CH}_{2} \mathrm{OBn}\right), 61.1\left(\mathrm{CO}_{2} \mathrm{CH}_{2} \mathrm{CH}_{3}\right), 52.8$ $\left(\mathrm{CO}_{2} \mathrm{Me}\right), \quad 36.9$ and $36.4\left(\mathrm{CH}_{2} \mathrm{C}\left(=\mathrm{CH}_{2}\right) \mathrm{CH}_{2}\right), 30.2\left(\mathrm{CH}_{2} \mathrm{C}(\mathrm{OH})\right), 25.9$ $\left(\mathrm{SiC}\left(\mathrm{CH}_{3}\right)_{3}\right), 18.6\left(\mathrm{SiC}\left(\mathrm{CH}_{3}\right)_{3}\right), 14.6\left(\mathrm{CO}_{2} \mathrm{CH}_{2} \mathrm{CH}_{3}\right),-3.4$ and $-3.08(2 \mathrm{x}$ $\left.\mathrm{SiCH}_{3}\right)$.

HRMS $m / z\left(\mathrm{M}+\mathrm{H}^{+}\right)$found: 505.2729. $\mathrm{C}_{26} \mathrm{H}_{41} \mathrm{O}_{6} \mathrm{~N}_{2}{ }^{28} \mathrm{Si}$ requires 505.2728.

Second eluted cycloadduct 23a (14 mg, 37\%), as a pale yellow oil; $R_{f}=$ $0.34\left(20 \% \mathrm{Et}_{2} \mathrm{O}\right.$ in petrol).

$v_{\max } / \mathrm{cm}^{-1}$ (film) $2928 \mathrm{~m}, 2855 \mathrm{~m}, 1741 \mathrm{~s}, 1454 \mathrm{~m}, 1365 \mathrm{w}, 1313 \mathrm{~m}, 1253$ s, $1100 \mathrm{~m}, 1056 \mathrm{~m}, 838 \mathrm{~s}, 779 \mathrm{~m}$.

${ }^{1} \mathrm{H}$ NMR (500 MHz; $\left.\mathrm{CDCl}_{3}\right) \delta 7.38-7.27(5 \mathrm{H}, \mathrm{m}, \mathrm{ArH}), 4.73(1 \mathrm{H}, \mathrm{d}, J$ 4.5, $\mathrm{CH} H \mathrm{~N}=\mathrm{N}), 4.52-4.43\left(3 \mathrm{H}, \mathrm{m}, \mathrm{CH}_{2} \mathrm{Ph}\right.$ and $\left.\mathrm{CH} H \mathrm{~N}=\mathrm{N}\right), 4.17(2 \mathrm{H}, \mathrm{qd}, J 7$ and 2, $\left.\mathrm{CO}_{2} \mathrm{CH}_{2} \mathrm{CH}_{3}\right), 3.79$ (3H, s, $\left.\mathrm{CO}_{2} \mathrm{Me}\right), 3.57-3.48\left(2 \mathrm{H}, \mathrm{m}, \mathrm{CH}_{2} \mathrm{OBn}\right), 2.41-$ $2.32(1 \mathrm{H}, \mathrm{m}, \mathrm{CH} H \mathrm{C}(\mathrm{OTBS})), 2.09-2.02\left(1 \mathrm{H}, \mathrm{m}, \mathrm{CH}_{\mathrm{CH}} \mathrm{C}(\mathrm{OTBS})\right), 1.88-$ $1.78\left(2 \mathrm{H}, \mathrm{m}, \mathrm{CH} \mathrm{HC}(\mathrm{OTBS})\right.$ and $\left.\mathrm{CHHCH}_{2} \mathrm{OBn}\right), 1.65-1.60(1 \mathrm{H}, \mathrm{m}$, $\left.\mathrm{CHHCH}_{2} \mathrm{OBn}\right), 1.57-1.50\left(1 \mathrm{H}, \mathrm{m}, \mathrm{CHHCH}_{2} \mathrm{C}(\mathrm{OTBS})\right), 1.25(3 \mathrm{H}, \mathrm{t}, J \mathrm{~J}$, $\mathrm{CO}_{2} \mathrm{CH}_{2} \mathrm{CH}_{3}$ ), $0.82\left(9 \mathrm{H}, \mathrm{s}, \mathrm{OSiCMe}_{3}\right), 0.05\left(6 \mathrm{H}, 2 \mathrm{x} \mathrm{s}, \mathrm{OSi}\left(\mathrm{CH}_{3}\right)_{2}\right)$.

${ }^{13} \mathrm{C}$ NMR (125 MHz; $\left.\mathrm{CDCl}_{3}\right) \delta 172.0\left(\mathrm{CO}_{2} \mathrm{Me}\right), 168.2\left(\mathrm{CO}_{2} \mathrm{Et}\right), 138.1(\mathrm{ArC})$, $128.6(\mathrm{ArCH}), 127.9(\mathrm{ArCH}), 127.7(\mathrm{ArCH}), 110.5$ (quat. $\left.\mathrm{CCO}_{2} \mathrm{Et}\right), 90.9$ $\left(\mathrm{CH}_{2} \mathrm{~N}=\mathrm{N}\right), 87.1$ (quat. $\left.C(\mathrm{OTBS}) \mathrm{CO}_{2} \mathrm{Me}\right), 73.4\left(\mathrm{CH}_{2} \mathrm{Ph}\right), 67.8\left(\mathrm{CH}_{2} \mathrm{OBn}\right)$, $61.9\left(\mathrm{CO}_{2} \mathrm{CH}_{2} \mathrm{CH}_{3}\right), \quad 52.3 \quad\left(\mathrm{CO}_{2} \mathrm{Me}\right), 49.6 \quad$ (quat. $\left.\quad \mathrm{CCH}_{2} \mathrm{~N}=\mathrm{N}\right), \quad 37.4$ ( $\left.\mathrm{CH}_{2} \mathrm{C}(\mathrm{OTBS})\right), \quad 35.3 \quad\left(\mathrm{CH}_{2} \mathrm{CH}_{2} \mathrm{OBn}\right), \quad 34.5 \quad\left(\mathrm{CH}_{2} \mathrm{CH}_{2} \mathrm{C}(\mathrm{OTBS})\right), \quad 25.7$ $\left(\mathrm{SiC}\left(\mathrm{CH}_{3}\right)_{3}\right), 18.5\left(\mathrm{SiC}\left(\mathrm{CH}_{3}\right)_{3}\right), 14.2\left(\mathrm{CO}_{2} \mathrm{CH}_{2} \mathrm{CH}_{3}\right),-3.1$ and $-4.0(2 \mathrm{x}$ $\left.\mathrm{SiCH}_{3}\right)$.

HRMS $m / z\left(\mathrm{M}+\mathrm{H}^{+}\right)$found: $505.2732 . \mathrm{C}_{26} \mathrm{H}_{41} \mathrm{O}_{6} \mathrm{~N}_{2}{ }^{28} \mathrm{Si}$ requires 505.2728 . 
4-Ethyl 1-methyl 2-(5-(benzyloxy)-3-methylenepentyl)-3-diazo-2((trimethylsilyl)oxy) succinate (22b) and 6a-Ethyl 6-methyl (3aR, $6 S$, 6aR)-3a-(2-(benzyloxy)ethyl)-6-((trimethylsilyl)oxy)-3a,4,5,6tetrahydrocyclopenta[c]pyrazole-6,6a(3H)-dicarboxylate $(23 \mathrm{~b})$

Hexamethyldisilazane $(66 \mu \mathrm{l}, 0.32 \mathrm{mmol})$ was added to a solution of diazo-alcohol 21 (30 mg, $0.076 \mathrm{mmol}$ ) and imidazole (11 mg, $0.16 \mathrm{mmol}$ ) in THF $(0.8 \mathrm{ml})$ at rt. After $48 \mathrm{~h}$, the reaction mixture was concentrated by blowing nitrogen, followed by purification of the residue by column chromatography $\left(0-10 \% \mathrm{Et}_{2} \mathrm{O}\right.$ in petrol).

First eluted TMS ether 22b (14 mg, 40\%), as a yelow oil; $R_{f}=0.26(20 \%$ $\mathrm{Et}_{2} \mathrm{O}$ in petrol).

$v_{\max } / \mathrm{cm}^{-1}$ (film) $2954 \mathrm{~m}, 2096 \mathrm{~s}, 1744 \mathrm{~s}, 1701 \mathrm{~s}, 1454 \mathrm{~m}, 1369 \mathrm{~m}, 1304 \mathrm{~s}$, $1251 \mathrm{~s}, 1135 \mathrm{~m}, 844 \mathrm{~s}$.

${ }^{1} \mathrm{H}$ NMR (400 MHz; $\mathrm{CDCl}_{3}$ ) $\delta$ 7.37-7.27 (5H, m, $\mathrm{Ar} H$ ), $4.80\left(2 \mathrm{H}, \mathrm{s}, \mathrm{C}=\mathrm{CH}_{2}\right.$ ), $4.51\left(2 \mathrm{H}, \mathrm{s}, \mathrm{CH}_{2} \mathrm{Ph}\right), 4.26-4.11\left(2 \mathrm{H}, \mathrm{m}, \mathrm{CO}_{2} \mathrm{CH}_{2} \mathrm{CH}_{3}\right), 3.73\left(3 \mathrm{H}, \mathrm{s}, \mathrm{CO}_{2} \mathrm{Me}\right)$ $3.56\left(2 \mathrm{H}, \mathrm{t}, J \mathrm{~J}, \mathrm{CH} \mathrm{CHBB}_{2} \mathrm{O}, 2.34\left(2 \mathrm{H}, \mathrm{t}, J \mathrm{~J}, \mathrm{CH}_{2} \mathrm{CH}_{2} \mathrm{OBn}\right), 2.22-1.92(4 \mathrm{H}, \mathrm{m}\right.$, $\mathrm{CH}_{2} \mathrm{CH}_{2} \mathrm{C}(\mathrm{OTBS})$ ), 1.25 (3H, t, J 7, $\mathrm{CO}_{2} \mathrm{CH}_{2} \mathrm{CH}_{3}$ ), 0.13 (9H, s, OTMS)

${ }^{13} \mathrm{C}$ NMR (100 MHz; $\left.\mathrm{CDCl}_{3}\right) \delta 171.8\left(\mathrm{CO}_{2} \mathrm{Me}\right), 164.9\left(\mathrm{CO}_{2} \mathrm{Et}\right), 145.6$ $\left(\mathrm{C}=\mathrm{CH}_{2}\right), 138.5(\mathrm{ArC}), 128.5(\mathrm{ArCH}), 127.8(\mathrm{ArCH}), 127.7(\mathrm{ArCH}), 111.1$ $\left(\mathrm{C}=\mathrm{CH}_{2}\right), 76.2$ (quat. $\left.C(\mathrm{OTMS}) \mathrm{CO}_{2} \mathrm{Me}\right), 73.1\left(\mathrm{CH}_{2} \mathrm{Ph}\right), 68.9\left(\mathrm{CH}_{2} \mathrm{OBn}\right), 61.0$ $\left(\mathrm{CO}_{2} \mathrm{CH}_{2} \mathrm{CH}_{3}\right), 52.8\left(\mathrm{CO}_{2} \mathrm{Me}\right), 36.7$ and $36.5\left(\mathrm{CH}_{2} \mathrm{C}\left(=\mathrm{CH}_{2}\right) \mathrm{CH}_{2}\right), 30.2$ ( $\left.\mathrm{CH}_{2} \mathrm{C}(\mathrm{OTMS})\right), 14.6\left(\mathrm{CO}_{2} \mathrm{CH}_{2} \mathrm{CH}_{3}\right), 1.4$ (OTMS).

HRMS $m / z\left(\mathrm{M}+\mathrm{H}^{+}\right)$found: 463.2258. $\mathrm{C}_{23} \mathrm{H}_{35} \mathrm{O}_{6} \mathrm{~N}_{2}{ }^{28}$ Si requires 463.2258 .

Second eluted cycloadduct $\mathbf{2 3 b}\left(11 \mathrm{mg}, 31 \%\right.$ ), as a pale yellow oil; $R_{f}=$ $0.34\left(20 \% \mathrm{Et}_{2} \mathrm{O}\right.$ in petrol).

$v_{\max } / \mathrm{cm}^{-1}$ (film) $2951 \mathrm{~m}, 2928 \mathrm{~m}, 1741$ s, 1455 m, 1254 s, 1101 m, 1056 m, $839 \mathrm{~s}, 779 \mathrm{~m}$.

${ }^{1} \mathrm{H}$ NMR (500 MHz; $\left.\mathrm{CDCl}_{3}\right) \delta$ 7.38-7.27 (5H, m, ArH), $4.72(1 \mathrm{H}, \mathrm{d}, J$ 18.5, $\mathrm{CH} H \mathrm{~N}=\mathrm{N}), 4.53-4.42\left(3 \mathrm{H}, \mathrm{m}, \mathrm{CH}_{2} \mathrm{Ph}\right.$ and $\left.\mathrm{CH} H \mathrm{~N}=\mathrm{N}\right), 4.17(2 \mathrm{H}, \mathrm{qd}, J 7$ and 4, $\left.\mathrm{CO}_{2} \mathrm{CH}_{2} \mathrm{CH}_{3}\right), 3.80\left(3 \mathrm{H}, \mathrm{s}, \mathrm{CO}_{2} \mathrm{Me}\right), 3.56-3.47\left(2 \mathrm{H}, \mathrm{m}, \mathrm{CH}_{2} \mathrm{OBn}\right), 2.33(1 \mathrm{H}$, $\mathrm{dt}, J 13.5$ and 7.5, CHHC(OTBS)), 2.12-2.04 (1H, m, $\mathrm{CHHCH}_{2} \mathrm{C}(\mathrm{OTBS})$ ), $1.85\left(1 \mathrm{H}, \mathrm{dt}, J 13.5\right.$ and 5.5, $\left.\mathrm{CH} \mathrm{CH}_{2} \mathrm{OBn}\right), 1.75(1 \mathrm{H}, \mathrm{ddd}, J 13.5,7.5$ and 6.5, CHHC(OTBS)), 1.64-1.52 (1H, m, $\mathrm{CHHCH}_{2} \mathrm{OBn}$ and $\mathrm{CHHCH}_{2} \mathrm{C}(\mathrm{OTBS})$ ), $1.24\left(3 \mathrm{H}, \mathrm{t}, J 7, \mathrm{CO}_{2} \mathrm{CH}_{2} \mathrm{CH}_{3}\right), 0.10\left(9 \mathrm{H}, \mathrm{s}, \mathrm{OSi}\left(\mathrm{CH}_{3}\right)_{3}\right)$.

${ }^{13} \mathrm{C}$ NMR (125 MHz; $\left.\mathrm{CDCl}_{3}\right) \delta 172.4\left(\mathrm{CO}_{2} \mathrm{Me}\right), 167.8\left(\mathrm{CO}_{2} \mathrm{Et}\right), 138.2(\mathrm{ArC})$, 128.6 $(\mathrm{ArCH}), 127.8(\mathrm{ArCH}), 127.7(\mathrm{ArCH}), 110.1$ (quat. $\left.\mathrm{CCO}_{2} \mathrm{Et}\right), 91.9$ $\left(\mathrm{CH}_{2} \mathrm{~N}=\mathrm{N}\right), 87.0$ (quat. $\left.C(\mathrm{OTBS}) \mathrm{CO}_{2} \mathrm{Me}\right), 73.4\left(\mathrm{CH}_{2} \mathrm{Ph}\right), 67.7\left(\mathrm{CH}_{2} \mathrm{OBn}\right)$, $61.8\left(\mathrm{CO}_{2} \mathrm{CH}_{2} \mathrm{CH}_{3}\right), \quad 52.3 \quad\left(\mathrm{CO}_{2} M e\right), 49.6$ (quat. $\left.\quad C_{C H} \mathrm{~N}=\mathrm{N}\right), \quad 37.5$ ( $\left.\mathrm{CH}_{2} \mathrm{C}(\mathrm{OTBS})\right), \quad 35.5 \quad\left(\mathrm{CH}_{2} \mathrm{CH}_{2} \mathrm{OBn}\right), \quad 34.8 \quad\left(\mathrm{CH}_{2} \mathrm{CH}_{2} \mathrm{C}(\mathrm{OTBS})\right), \quad 14.2$ $\left(\mathrm{CO}_{2} \mathrm{CH}_{2} \mathrm{CH}_{3}\right), 1.6\left(\mathrm{Si}\left(\mathrm{CH}_{3}\right)_{3}\right)$.

HRMS $m / z\left(\mathrm{M}+\mathrm{H}^{+}\right)$found: 463.2251. $\mathrm{C}_{23} \mathrm{H}_{35} \mathrm{O}_{6} \mathrm{~N}_{2}{ }^{28}$ Si requires 463.2258.

\section{4-Ethyl 1-methyl 2-(5-(benzyloxy)-3-oxopentyl)-2-((tert-butyl} dimethylsilyl)oxy)-3-diazosuccinate (24a)

Following the general procedure for ozonolysis above, using TBS ether 22a ( $4 \mathrm{mg}, 0.008 \mathrm{mmol}$ ), gave after column chromatography $(0-40 \%$ $\mathrm{Et}_{2} \mathrm{O}$ in petrol) diazoketone $\mathbf{2 4 a}(2.3 \mathrm{mg}, 58 \%)$, as a yellow oil; $R_{f}=0.53$ (50\% $\mathrm{Et}_{2} \mathrm{O}$ in petrol).

$v_{\max } / \mathrm{cm}^{-1}$ (film) $2931 \mathrm{~m}, 2856 \mathrm{~m}, 2095 \mathrm{~s}, 1747 \mathrm{~s}, 1700 \mathrm{~s}, 1456 \mathrm{w}, 1305 \mathrm{~m}$ $1256 \mathrm{~m}, 1132 \mathrm{~m}, 838 \mathrm{~m}$.

${ }^{1} \mathrm{H}$ NMR (500 MHz; $\left.\mathrm{CDCl}_{3}\right) \delta$ 7.37-7.27 (5H, m, $\left.\mathrm{ArH}\right), 4.50\left(2 \mathrm{H}, \mathrm{s}, \mathrm{CH}_{2} \mathrm{Ph}\right)$, 4.24-4.12 (2H, m, $\left.\mathrm{CO}_{2} \mathrm{CH}_{2} \mathrm{CH}_{3}\right), 3.75-3.68$ (5H, m, $\mathrm{CO}_{2} \mathrm{Me}$ and $\mathrm{CH}_{2} \mathrm{OBn}$ ), 2.69 (2H, t, J 6, $\mathrm{CH}_{2} \mathrm{CH}_{2} \mathrm{OBn}$ ), 2.67-2.61 (1H, m, CHHC(OTBS)), 2.51-2.44 (1H, m, CHHC(OTBS)), 2.27-2.21 (2H, m, $\left.\mathrm{CH}_{2} \mathrm{CH}_{2} \mathrm{C}(\mathrm{OTBS})\right), 1.24(3 \mathrm{H}, \mathrm{t}, J$ 7, $\left.\mathrm{OCH}_{2} \mathrm{CH}_{3}\right), 0.86(9 \mathrm{H}, \mathrm{s}, \mathrm{OSiCMe} 3), 0.09$ and $0.04\left(6 \mathrm{H}, 2 \mathrm{x} \mathrm{s}, \mathrm{OSi}\left(\mathrm{CH}_{3}\right)_{2}\right)$.

${ }^{13} \mathrm{C}$ NMR (125 MHz; $\left.\mathrm{CDCl}_{3}\right) \delta 207.7(\mathrm{C}=0), 171.4\left(\mathrm{CO}_{2} \mathrm{Me}\right), 164.8\left(\mathrm{CO}_{2} \mathrm{Et}\right)$, $138.2(\mathrm{ArC}), 128.5(\mathrm{ArCH}), 127.8(\mathrm{ArCH}), 127.8(\mathrm{ArCH}), 75.6$ (quat. $\left.C(\mathrm{OTBS}) \mathrm{CO}_{2} \mathrm{Me}\right), 73.4\left(\mathrm{CH}_{2} \mathrm{Ph}\right), 65.4\left(\mathrm{CH}_{2} \mathrm{OBn}\right), 61.2\left(\mathrm{CO}_{2} \mathrm{CH}_{2} \mathrm{CH}_{3}\right), 52.9$ $\left(\mathrm{CO}_{2} \mathrm{Me}\right), 43.2\left(\mathrm{O}=\mathrm{C}-\mathrm{CH}_{2}\right), 37.8\left(\mathrm{O}=\mathrm{C}-\mathrm{CH}_{2}\right), 32.2\left(\mathrm{CH}_{2} \mathrm{C}(\mathrm{OTBS})\right), 25.9$ $\left(\mathrm{SiC}\left(\mathrm{CH}_{3}\right)_{3}\right), 18.6\left(\mathrm{SiC}\left(\mathrm{CH}_{3}\right)_{3}\right), 14.6\left(\mathrm{CO}_{2} \mathrm{CH}_{2} \mathrm{CH}_{3}\right),-3.5$ and $-3.9(2 \mathrm{x}$ $\left.\mathrm{SiCH}_{3}\right)$.

HRMS $m / z \quad\left(M+\mathrm{Na}^{+}\right)$found: 529.2341. $\mathrm{C}_{25} \mathrm{H}_{38} \mathrm{O}_{7} \mathrm{~N}_{2}{ }^{23} \mathrm{Na}^{28} \mathrm{Si}$ requires 529.2340 .
4-Ethyl 1-methyl 2-(5-(benzyloxy)-3-oxopentyl)-3-diazo-2-((tri methylsilyl)oxy)succinate (24b)

Following the procedure for ozonolysis above, using TMS ether 22b (6 $\mathrm{mg}, 0.013 \mathrm{mmol})$, gave after column chromatography $\left(0-40 \% \mathrm{Et}_{2} \mathrm{O}\right.$ in petrol) diazoketone $24 \mathbf{b}$ (3.7 mg, 61\%), as a yellow oil; $R_{f}=0.51(50 \%$ $\mathrm{Et}_{2} \mathrm{O}$ in petrol).

$v_{\max } / \mathrm{cm}^{-1}$ (film) $2953 \mathrm{w}, 2871 \mathrm{w}, 2096 \mathrm{~s}, 1747 \mathrm{~s}, 1702$ s, $1455 \mathrm{w}, 1369 \mathrm{~m}$, $1305 \mathrm{~s}, 1251 \mathrm{~s}, 1135 \mathrm{~m}, 845 \mathrm{~s}$.

${ }^{1} \mathrm{H}$ NMR (500 MHz; $\left.\mathrm{CDCl}_{3}\right) \delta$ 7.37-7.27 (5H, m, $\mathrm{Ar} H$ ), $4.50\left(2 \mathrm{H}, \mathrm{s}, \mathrm{CH}_{2} \mathrm{Ph}\right)$, 4.27-4.11 (2H, m, $\left.\mathrm{CO}_{2} \mathrm{CH}_{2} \mathrm{CH}_{3}\right), 3.75-3.71\left(5 \mathrm{H}, \mathrm{m}, \mathrm{CO}_{2} \mathrm{Me}\right.$ and $\left.\mathrm{CH}_{2} \mathrm{OBn}\right)$, 2.70 (2H, t, J 6.5, $\mathrm{CH}_{2} \mathrm{CH}_{2} \mathrm{OBn}$ ), 2.66-2.58 (1H, m, CHHC(OTMS)), 2.482.41 (1H, m, CHHC(OTMS)), 2.26-2.20 (2H, m, $\left.\mathrm{CH}_{2} \mathrm{CH}_{2} \mathrm{C}(\mathrm{OTMS})\right), 1.25$ $\left(3 \mathrm{H}, \mathrm{t}, J 7, \mathrm{OCH}_{2} \mathrm{CH}_{3}\right), 0.11$ (3H, s, OTMS).

${ }^{13} \mathrm{C}$ NMR (125 MHz; $\left.\mathrm{CDCl}_{3}\right) \delta 207.8(\mathrm{C}=0), 171.5\left(\mathrm{CO}_{2} \mathrm{Me}\right), 164.8\left(\mathrm{CO}_{2} \mathrm{Et}\right)$, $138.2(\mathrm{ArC}), 128.6(\mathrm{ArCH}), 127.8(\mathrm{ArCH}), 127.8(\mathrm{ArCH}), 75.8$ (quat. $C$ (OTMS $\left.) \mathrm{CO}_{2} \mathrm{Me}\right), 73.4\left(\mathrm{CH}_{2} \mathrm{Ph}\right), 65.4\left(\mathrm{CH}_{2} \mathrm{OBn}\right), 61.2\left(\mathrm{CO}_{2} \mathrm{CH}_{2} \mathrm{CH}_{3}\right), 53.0$ $\left(\mathrm{CO}_{2} \mathrm{Me}\right), 43.2\left(\mathrm{O}=\mathrm{C}-\mathrm{CH}_{2}\right), 37.7\left(\mathrm{O}=\mathrm{C}-\mathrm{CH}_{2}\right), 32.2\left(\mathrm{CH}_{2} \mathrm{C}(\mathrm{OTMS})\right), 14.6$ $\left(\mathrm{CO}_{2} \mathrm{CH}_{2} \mathrm{CH}_{3}\right), 1.3$ (OTMS).

HRMS $m / z \quad\left(\mathrm{M}+\mathrm{Na}^{+}\right)$found: 487.1867. $\mathrm{C}_{22} \mathrm{H}_{32} \mathrm{O}_{7} \mathrm{~N}_{2}{ }^{23} \mathrm{Na}^{28} \mathrm{Si}$ requires 487.1871.

\section{Funding Information}

We thank the Higher Committee for Education Development in Iraq, the EPSRC and the Higher Education Commission of Pakistan for studentship support (to H. A. A. A., Y.F.H. and T.A., respectively).

\section{Supporting Information}

YES (this text will be updated with links prior to publication)

\section{References and Notes}

(1) Permanent address: University of Kufa, Najaf Governorate, Iraq.

(2) Fegheh-Hassanpour, Y.; Arif, T.; Sintim, H. O.; Al-Mamari, H. A.; Hodgson, D. M. Org. Lett., 2017, 19, 3540; corrigendum, 2018, 20 , 5528.

(3) Almohseni, H. A. A.; Al-Mamari, H. A.; Valade, A.; Sintim, H. O.; Hodgson, D. M. Chem. Commun., 2018, 52, 5354.

(4) Hodgson, D. M.; Labande, A. H.; Muthusamy, S. Org. React., 2013, 80, 133.

(5) For intramolecular cycloadditions between diazo and alkene functionality, see: (a) Padwa, A.; Ku, H. J. Org. Chem., 1980, 45, 3756; (b) Dauben, W. G.; Hendricks, R. T.; Luzzio, M. J.; Ng, H. P. Tetrahedron Lett., 1990, 31, 6969; (c) Jung, M. E.; Huang, A. Org. Lett., 2000, 2, 2659; (d) Taber, D. F.; Guo, P. J. Org. Chem., 2008, 73, 9479; (e) Liu, H.; O'Connor, M. J.; Sun, C.; Wink, D. J.; Lee, D. Org. Lett., 2013, 15, 2974; (f) Barroso, R.; Escribano, M.; Cabal, M.P.; Valdés, C. Eur. J. Org. Chem., 2014, 1672; (g) Xia, Y.; Wang, J. Chem. Soc. Rev., 2017, 46, 2306. See also: (h) Padwa, A.; Kulkarni, Y. S.; Zhang, Z. J. Org. Chem., 1990, 55, 4144.

(6) Fegheh-Hassanpour, Y.; Ebrahim, F.; Arif, T.; Sintim, H. O.; Claridge, T. D. W.; Amin, N. T.; Hodgson, D. M. Org. Biomol. Chem., 2018, 16, 2876.

(7) (a) House, H. O.; Blankley, C. J. J. Org. Chem., 1968, 33, 53; (b) Blankley, C. J.; Sauter, F. J.; House, H. O. Org. Synth. Coll. Vol. V; John Wiley \& Sons: London, 1973, 258.

(8) For examples of ozonolysis of diazo compounds to give ketones, see: (a) Erickson, R. E.; Andrulis Jr., P. J.; Collins, J. C.; Lungle, M. L.; Mercer, G. D. J. Org. Chem., 1969, 34, 2961; (b) Sekiguchi, A.; Ando, W. J. Chem. Soc., Chem. Commun., 1979, 575; (c) Ursini, A.; Pellicciari, R.; Tamburini, B.; Carlesso, R.; Gaviraghi, G. Synthesis, 1992, 363.

(9) (a) Schöllkopf, U.; Frasnelli, H. Angew. Chem., Int. Ed. Engl., 1970, 9, 301; (b) Wenkert, E.; McPherson, C. A. J. Am. Chem. Soc., 1972, 94,8084 . 
(10) Hon and co-workers (Hon, Y.-S.; Lin, S.-W.; Lu, L.; Chen, Y.-J. Tetrahedron, 1995, 51, 5019) report that, for ozonolysis of alkenes (eg, 2,2-disubstituted-1-alkenes), Et ${ }_{3} \mathrm{~N}$ typically gives better yields and proceeds faster compared to $\mathrm{Me}_{2} \mathrm{~S}$. $\mathrm{Me}_{2} \mathrm{~S}$ also proved viable in the current transformation, but the crude reaction mixture was not as clean as with $\mathrm{Et}_{3} \mathrm{~N}$.

(11) (a) Poschenrieder, H.; Stachel, H.-D. Arch. Pharm. (Weinheim, Ger.), 1989, 322, 301; (b) Hodgson, D. M.; Man, S. Chem. Eur. J., 2011, 17, 9731; (c) Hodgson, D. M.; Moreno-Clavijo, E.; Day, S. E.; Man, S. Org. Biomol. Chem., 2013, 11, 5362.

(12) Jiang, Y.; Khong, V. Z. Y.; Lourdusamy, E.; Park, C.-M. Chem. Commun., 2012, 48, 3133.

(13) (a) Eftekhari-Sis, B.; Zirak, M. Chem. Rev., 2015, 115, 151; (b) de las Heras, M. A.; Vaquero, J. J.; García-Navio, J. L.; Alvarez-Builla, J. J. Org. Chem., 1996, 61, 9009; (c) Weinstock, L. M.; Currie, R. B.; Lovell, A. V. Synth. Commun., 1981, 11, 943.
(14) For examples, see: (a) Macritchie, J. A.; Silcock, A.; Willis, C. L. Tetrahedron: Asymmetry, 1997, 8, 3895; (b) Vickers, T. D.; Keay, B. A. Synlett, 2003, 1349.

(15) Bailey, W. F.; Punzalan, E. R. J. Org. Chem., 1990, 55, 5404.

(16) On a similar motif, $\gamma$-lactolisation of a $\beta$-hydroxy- $\varepsilon$-oxo- $\alpha$ diazoester was observed: Villalonga-Barber, C. D Phil Thesis, University of Oxford, 2002.

(17) The stereochemistry of pyrazolines 23a,b were supported by NOESY experiments.

(18) Pangborn, A. B.; Giardello, M. A.; Grubbs, R. H.; Rosen, R. K.; Timmers, F. J. Organometallics, 1996, 15, 1518.

(19) Peng, C.; Wang, Y.; Wang, J. J. Am. Chem. Soc., 2008, 130, 1566.

(20) Siu, J. C.; Parry, J. B.; Lin, S. J. Am. Chem. Soc., 2019, 141, 2825. 